
\title{
$\begin{array}{ll}\text { Research Square } & \begin{array}{l}\text { Preprints are preliminary reports that have not undergone peer review. } \\ \text { They should not be considered conclusive, used to inform clinical practice, } \\ \text { or referenced by the media as validated information. }\end{array}\end{array}$
}

\section{D dynamic tractography visualizes the effective connectivity supporting specific linguistic stages in the developing brain}

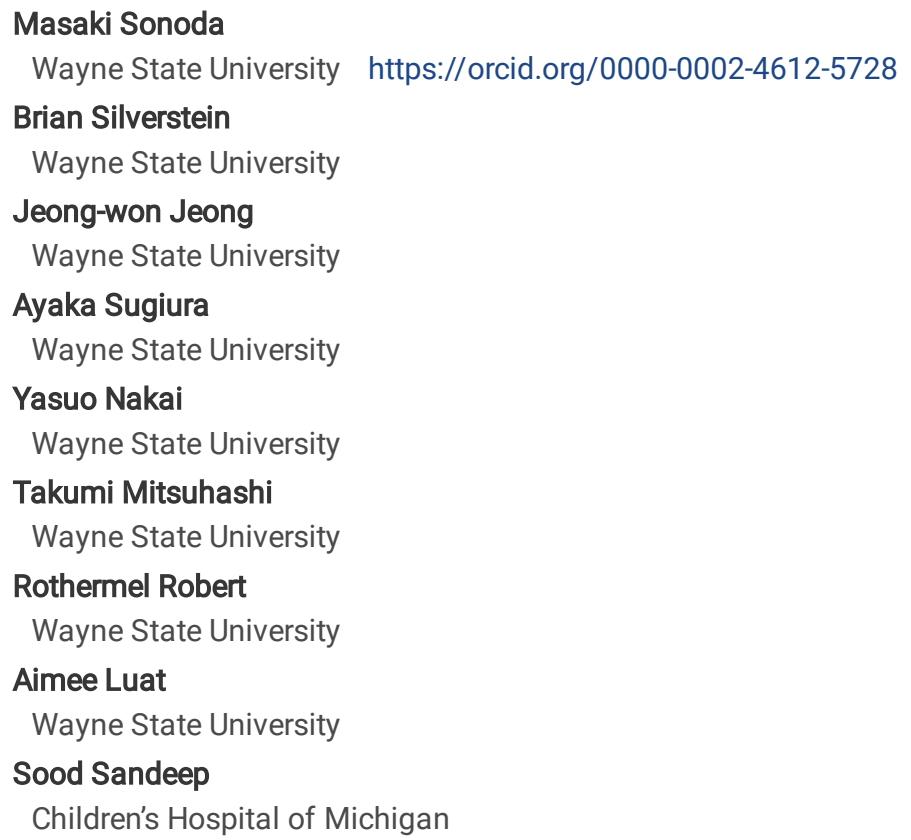

Keywords: high-gamma activity (HGA), cortico-cortical evoked potentials (CCEPs), intracranial electroencephalography (iEEG), Dynamic DWI tractography, pediatric epilepsy surgery.

Posted Date: February 2nd, 2021

DOI: https://doi.org/10.21203/rs.3.rs-150278/v1

License: (c) (i) This work is licensed under a Creative Commons Attribution 4.0 International License. Read Full License 


\section{Abstract}

During a verbal conversation, as individuals listen and respond, the human brain moves through a series of complex linguistic processing stages: decoding of speech sounds, semantic comprehension, retrieval of semantically coherent words, and finally, overt production of speech outputs. Each process is thought to be supported by a cortical network consisting of local and long-range connections bridging between major cortical areas. Both temporal and extratemporal lobe regions are suggested to have functional compartments responsible for distinct language domains, including the perception and production of phonological and semantic components. This study provides quantitative evidence of how directly connected, inter-lobar neocortical networks support distinct linguistic stages of linguistic processing across brain development. A novel six-dimensional tractography animation technique was used to intuitively visualize the strength and temporal dynamics of direct inter-lobar effective connectivity between cortical areas activated during distinct linguistic processing stages. This study analyzed 3,401 non-epileptic intracranial electrode sites from 37 children with focal epilepsy (age: 5-20 years) who underwent extraoperative electrocorticography recording. We used a principal component analysis of high-gamma modulations during an auditory naming task to determine the relative involvement of each cortical area during each linguistic processing stage. To quantify direct effective connectivity, we delivered single-pulse electrical stimulation to 488 temporal lobe sites and 1,581 extratemporal lobe sites and measured the early cortico-cortical spectral responses at distant electrodes. Mixed model analyses determined the effects of naming-related high-gamma co-augmentation between connecting regions, age, and cerebral hemisphere on the strength of effective connectivity independent of epilepsy-related factors. Direct effective connectivity was strongest between temporal and extratemporal lobe site pairs which were simultaneously activated during the period between sentence offset and verbal response onset (i.e., semantic retrieval period); this connectivity was approximately twice more robust than that with temporal lobe sites activated during stimulus listening or overt response. Conversely, extratemporal lobe sites activated during overt response were equally connected with temporal lobe language sites. Older age was associated with the increased strength of inter-lobar effective connectivity between those activated during semantic retrieval. The arcuate fasciculus supported approximately two-thirds of the direct effective connectivity pathways from temporal to extratemporal auditory language-related areas but only up to half of those in the opposite direction. The uncinate fasciculus consisted of only less than $2 \%$ of those in the temporal-to-extratemporal direction and up to $6 \%$ of those in the opposite direction. Our multimodal study quantified and animated the direct inter-lobar networks toward and from temporal lobe regions supporting distinct stages of auditory language processing. Additionally, age-dependent strengthening of connectivity after age five may preferentially occur between language areas supporting semantic retrieval.

\section{Introduction}

Human verbal conversations can be considered in two phases: reception and expression. Reception happens in two stages: initially, each sound in the heard sentence is recognized and decoded via phonological processing, followed by integration with higher-order functions to understand the semantic meaning ${ }^{1-5}$. The second phase, expression, also consists of two parts. Semantically coherent words are retrieved, followed by overt speech production and monitoring of one's own speech ${ }^{5-8}$. This complex, the multi-stage process is believed to be supported by a broad cortical network incorporating a combination of local processing and long-range inter-lobar connections. The precise spatial configuration and temporal dynamics of the language network are still active research, and multiple competing models exist ${ }^{2-4,9,10}$. Common among each model is evidence that long-range white matter tracts (e.g., the arcuate and uncinate fasciculi) from and toward the temporal lobe play a central role in the language network. These inter-lobar pathways are suggested to exchange language information, including mental representation of sentences, and facilitate the translation of sound and semantics into motor representations ${ }^{9-15}$. However, previous studies have not provided direct quantitative evidence of how much, how fast, via what fasciculus, and in which direction given inter-lobar fasciculi can transfer neural information between language areas supporting phonologic and semantic processes. Collective evidence also indicates that the development of inter-lobar connectivity networks begins early in life and continues in an experience-dependent manner, possibly beyond adolescence ${ }^{16,17}$. Investigators have not reached a consensus on the developmental patterns of effective connectivity between the temporal and extratemporal lobe regions supporting different linguistic processing stages.

This multimodality study aimed to localize, quantify, and visualize the strength and dynamics of effective connectivity networks that support direct transfers of neural activity between the temporal and extratemporal lobe neocortices supporting distinct linguistic processing stages. Investigators have proposed several models for how the cerebral cortex transfers neural representations of phonological and semantic information. The classical Geschwind model of language-related connectivity did not distinguish separate pathways supporting the phonological and semantic domains ${ }^{18}$. Instead, it proposed that the left posterior temporal lobe neocortex transfers the entire linguistic content expressed by a given spoken sentence to the left inferior frontal gyrus (IFG) for subsequent speech output. Conversely, most current models propose that neural activity representing phonological and semantic domains is transferred via different pathways and bihemispheric involvement, though with left-hemispheric dominance ${ }^{2-4,10}$. Some models suggest that the human brain transfers neural representations directly from the posterior temporal to the extratemporal lobe language areas, mainly via the arcuate fasciculus $\mathbf{s}^{3,4,10,11}$. Another model infers that auditory language information is transferred indirectly via another structure in the temporoparietal junction ${ }^{2}$. Another suggests that auditory semantic processing is initially supported by the neural information transfer from the left posterior to the anterior temporal area, which 
in turn projects to the inferior frontal region, presumably via the uncinate fasciculus ${ }^{9}$; subsequent semantic processing to understand auditory sentences is proposed to be supported by neural transfer from the inferior frontal to the posterior temporal region mainly via the arcuate fasiculus ${ }^{9}$.

The sound processing network is often referred to as the 'phonological loop.' It is localized mainly in the inferior Rolandic area, superior temporal gyrus (STG), and the surrounding regions of both hemispheres ${ }^{19-23}$. The 'phonological loop' is believed to support the transformation of perceptual to motor representations of spoken sounds and the monitoring of the volume/pitch of one's own spoken sounds $7,8,12,19$.

The semantic processing network comprises both temporal and extratemporal lobe regions, with left-hemispheric dominance, including the left IFG and inferior parietal region ${ }^{24-27}$. Linguistic processing is thought to be in part sequential ${ }^{5,28}$. Phonological process of an auditory input can begin once auditory information is received by the primary auditory cortex, followed by semantic understanding processes that continue until after the auditory stimulus offset. Following processing of a heard linguistic input, semantic retrieval begins before an individual starts preparing an overt response. Although to some degree each of these processes overlaps during verbal conversations ${ }^{28}$, the linear nature of language processing suggests that late neuronal engagement between the offset of auditory sentence stimuli and the onset of overt responses during an auditory naming task reflects, at least in part, semantic understanding and retrieval ${ }^{5,29}$. Interventional evidence from an intracranial study supports this notion; high-frequency electrical stimulation of left-hemispheric regions showing such late neuronal engagement often results in a transient impairment of semantic retrieval with intact phonological repetition ${ }^{5}$.

Electrocorticography (ECOG) recordings during cognitive tasks can provide detailed estimations of the spatiotemporal distribution of corresponding brain activity. In particular, event-related augmentation of broadband activity, including the high-gamma range at $70-110 \mathrm{~Hz}$, has been demonstrated to be an excellent surrogate marker for neural activation ${ }^{5,30-33}$. An increase in high-gamma amplitude is associated with increased firing rate on a single-neuron recording, hemodynamic response on functional MRI, and cortical metabolism on glucose-metabolism positron emission tomography ${ }^{34-36}$. The relevance of event-related high-gamma augmentation to language behavior has also been demonstrated. Naming-related high-gamma augmentation is capable of accurately predicting the language areas defined by electrical stimulation mapping (ESM) as well as postoperative language impairment ${ }^{37,38}$.

To quantify inter-lobar effective connectivity, we measured cortico-cortical spectral responses (CCSRs) elicited by weak single-pulse electrical stimulation at two adjacent electrode contacts ${ }^{39}$. While task-related high-gamma connectivity measures can demonstrate statistical covariation, they do not provide causal evidence of direct connectivity. Both CCSRs and cortico-cortical evoked potentials (CCEPs) are powerful tools to quantify the strength and dynamics of effective connectivity in each direction between two remote regions ${ }^{39-43}$. The two measures generally reflect the same underlying neuronal process but highlight different aspects of the recorded signal. CCSRs comprise a summation of phaselocked and asynchronous responses, whereas CCEPs consist of phase-locked responses alone. CCSRs are agnostic to the polarity of cortical responses, whereas CCEPs may exhibit a variable polarity of responses depending on the structural feature of the underlying cortex ${ }^{41}$. Previous studies suggest that early CCSRs roughly correspond to the early CCEP component (also known as N1), thus reflecting cortical excitation elicited by single-axonal neural propagation from the stimulus site $39,41,44-47$. This notion is supported by the observation that the peak latency of such early responses is correlated with the surface distance as well as the underlying white matter streamline length on diffusion-weighted imaging (DWI) tractography ${ }^{4-50}$. Late, low-frequency band CCSRs, roughly corresponding to the late CCEP component (also known as N2), are suggested to mainly indicate post-excitatory neuronal inhibition $39,44,46$ and represent an indirect measure of inhibitory networks that govern effective connectivity.

The present study utilized a novel multimodal approach, integrating ECoG high-gamma augmentation during an auditory naming task with CCSRs and DWI-based tractography to identify the strength, direction, and anatomical pathway of networks that support each stage of language processing. In this study, we sought to use the new perspective provided by an integrated, multimodal approach to test the spatiotemporal predictions of each proposed phonological and semantic network model ${ }^{19-23}$. To that end, for each stage of language processing, we quantified the strength and dynamics of the inter-lobar effective connectivity toward and from the temporal lobe and localized the corresponding white matter pathways. Auditory language-related cortical activity was thus measured using the dynamics of high-gamma amplitude modulations at each electrode site during an auditory naming task ${ }^{5}$. We then estimated the relative involvement of cortical areas in each linguistic processing stage with a principal component analysis (PCA) of the temporal variations in high-gamma amplitude modulations ${ }^{51}$.

Multimodal studies present a unique challenge for intuitive visualization and interpretation of results, which are often high-dimensional. Here, the spatiotemporal dynamics are represented in six dimensions: CCSR magnitude, CCSR dynamics, naming task-related high-gamma activity (HGA), and three spatial dimensions. To facilitate interpretation, we generated animations visualizing the strength and dynamics of effective connectivity (i.e., CCSR-based neural propagations) via white-matter tracts directly bridging cortical sites supporting specific linguistic processing stages. We refer to the multimodal analysis and corresponding animation-based atlas presented here as six-dimensional (6D)

Page $3 / 23$ 
dynamic tractography. We expected that our 6D dynamic tractography would validate or revise existing neurobiological models of language

organization and development ${ }^{2-4,9-11}$. We tested the specific hypothesis that extratemporal lobe sites supporting semantic retrieval would have direct effective connectivity toward and from temporal lobe sites supporting the same linguistic stage more robustly than phonological processing. We also quantitatively determined what specific fasciculi would support direct inter-lobar effective connectivity between given language sites in each direction. In this study of participants of age ranging between 5 and 20 years, we hypothesized that older age would increase the strength of inter-lobar effective connectivity specifically between those supporting the semantic rather than the phonological process.

\section{Materials And Methods}

\section{Participants}

We studied 37 patients with focal seizures (age: 5-20 years; Table 1and Supplementary Fig. S1) who underwent epilepsy surgery between February 2013 and August 2018 at Detroit Medical Center in Detroit, MI, USA, and satisfied the following criteria. The inclusion criteria included [i] age $\geq 4$ years $^{52}$ and [ii] extraoperative ECoG sampling from temporal and extratemporal lobe regions. The exclusion criteria consisted of [i] the presence of a massive structural lesion (such as megalencephaly or perisylvian polymicrogyria) that renders the landmarks for the central or lateral sulcus unidentifiable, [ii] an inability to complete the auditory naming task due to inadequate vocabulary, incomprehension of task instructions, or lack of cooperativity, [iii] history of previous epilepsy surgery, and [iv] right-hemispheric language dominance estimated based on left-handedness associated with an early epileptogenic lesion in the neocortex of the left hemisphere (the rationale has been discussed in ref. $\left.{ }^{38}\right)$.

Table 1

Patient profile.

\begin{tabular}{|c|c|}
\hline Number of patients & 37 \\
\hline Mean age (years) & 12.7 \\
\hline Range of age (years) & $5-20$ \\
\hline Proportion of male (\%) & 43.2 \\
\hline Proportion of right-handedness (\%) & 89.2 \\
\hline Proportion of sampled hemisphere (\%): left/right/bilateral & $45.9 / 48.6 / 5.4$ \\
\hline \multicolumn{2}{|l|}{$\mathrm{SOZ}, n(\%)$} \\
\hline Frontal & $10(27.0)$ \\
\hline Temporal & $16(43.2)$ \\
\hline Parietal & $11(29.7)$ \\
\hline Occipital & $6(16.2)$ \\
\hline $\mathrm{N} / \mathrm{A}$ * & $2(5.4)$ \\
\hline Mean number of antiepileptic drugs & 1.9 \\
\hline MRI-visible cortical lesion, $n(\%)$ & $24(64.9)$ \\
\hline \multicolumn{2}{|l|}{ Etiology, $n(\%)$} \\
\hline Tumor & $5(13.5)$ \\
\hline Focal cortical dysplasia & $12(32.4)$ \\
\hline Hippocampal sclerosis & $1(2.7)$ \\
\hline Non-lesional or gliosis alone & $20(54.1)$ \\
\hline Mean number of artifact-free non-epileptic electrodes & 87.3 \\
\hline
\end{tabular}


All procedures were performed as part of our routine presurgical evaluation, and the spatial extent and duration of extraoperative ECoG recording and electrical stimulation were determined based on clinical demands $20,41,53,54$. This study has been approved by the Wayne State University Institutional Review Board. Written informed consent was obtained from the legal parents or guardians of patients. Written assent was obtained from children older than 13 , and oral assent was obtained from younger children.

\section{Subdural electrode placement and 3D localization}

Each patient underwent placement of platinum grid and strip electrodes (10 mm center-to-center distance; $3 \mathrm{~mm}$ diameter contact) in the subdural space to localize the boundaries between the epileptogenic zone and eloquent areas. Electrode plates were anchored with sutures to adjacent plates or the dura mater's edge to avoid electrode movement after placement ${ }^{54}$.

We created a 3D surface image for each patient and determined each electrode's location on the cortical surface, using the preoperative T1 spoiled gradient-recalled MRI and the FreeSurfer software package (http://surfer.nmr.mgh.harvard.edu) ${ }^{5,55,56}$. We confirmed the spatial accuracy of the electrode display using an intraoperative photograph ${ }^{57}$. We then spatially normalized all individual electrode sites' location to

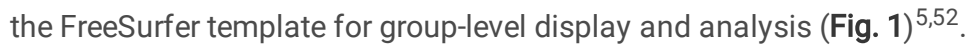

\section{Extraoperative ECoG recording}

ECoG was recorded at a sampling frequency of 1,000 Hz using a 192-channel Neurofax 1100A Digital System (Nihon Kohden America Inc., Foothill Ranch, CA, USA). To make our results more generalizable, we limited the quantitative analysis to non-epileptic electrode sites ${ }^{58,59}$. Electrode sites classified as seizure onset zone $(\mathrm{SOZ})^{53}$, those showing interictal spikes ${ }^{31,60}$, and those located on a structural lesion ${ }^{58}$ were excluded from the analysis. Likewise, electrodes affected by artifacts, including electromyographic ones, during the auditory naming task or the CCSR acquisition period were excluded from analysis ${ }^{54,61}$. After rejecting unusable channels, a total of 3,401 non-epileptic sites were included in the analysis. For both high-gamma and CCSR analyses, ECoG signals were analyzed using a common average reference (i.e., an average of signals across all non-artifactual, non-epileptic channels ${ }^{5450}$ ).

\section{Linguistic stage categorization using naming-related high-gamma activity}

Each patient was assigned a series of auditory question trials and instructed to verbally answer each question (Fig. 2). All question stimuli, starting with either 'what', 'where', 'when', or 'who', were sentences designed to draw out answers with nouns. Trials without correct answers were excluded from the analysis ${ }^{62}$. The mean rate of correct answers was $93.2 \pm 1.9 \%$ ( \pm standard error: SE). Response time was defined as the delay between sentence offset and response onset. The median response time across trials was $1.58 \pm 0.07$ seconds on average across patients.

At each electrode site, we quantified naming-related high-gamma $(70-110 \mathrm{~Hz})$ amplitude as a summary measure reflecting the magnitude of neuronal engagement at each moment during auditory verbal discourse (Fig. 2) 5,54 . ECoG signals were first transformed into the frequency domain using a complex demodulation technique implemented in BESA ${ }^{\circledR 囚}$ EEG V.5.1.8 (BESA GmbH, Gräfelfing, Germany) ${ }^{63,64}$ in nonoverlapping $10 \mathrm{~ms}$ time and $5 \mathrm{~Hz}$ frequency bins $5,38,65,66$. We computed high-gamma amplitude augmentation by averaging the amplitude within $70-110 \mathrm{~Hz}$ and normalizing to the mean high-gamma amplitude in a 600 to 200 ms pre-stimulus baseline period (Fig. 2a). We previously reported that sites showing naming-related high-gamma augmentation were spatially concordant with the language-related areas defined by $\mathrm{ESM}^{5,66}$. Furthermore, resection of sites showing naming-related high-gamma augmentation increased the risk of acute language deficits requiring speech therapy ${ }^{38}$.

The PCA transformed six 300 ms windows (Fig. 2b) into a new dimensional space of orthogonal components (Fig. 3a) ${ }^{51}$. The first principal components (PCs) explaining 95\% of the variance in high-gamma temporal dynamic patterns were extracted and used to categorize the linguistic processing stages (Fig. 3).

\section{Quantification of direct inter-lobar effective connectivity using early CCSRs}

We identified direct effective connectivity between temporal and extratemporal lobe electrode sites by measuring cortical responses to local single-pulse electrical stimulation 20,49 . As part of our routine presurgical evaluation $47,49,67$, we delivered trains of weak electrical stimuli to a total of 488 electrode sites (314 pairs of adjacent electrodes, 8.5 pairs per patient on average; Fig. 1b) within the temporal lobe neocortex (STG, middle temporal gyrus [MTG], and inferior temporal gyrus) and 1,581 extratemporal lobe electrode sites (885 pairs of adjacent electrodes, 24.6 pairs per patient on average; Fig. 1c) at a frequency of $1 \mathrm{~Hz}$ for $40 \mathrm{~s}$ during sleep. Each stimulus consisted of a biphasic square wave with a pulse width of $0.3 \mathrm{~ms}$ and an intensity of $5 \mathrm{~mA}$. The stimulus intensity was smaller than those previously reported to be safe ${ }^{40,42,68,69}$. No adverse events were noted during the CCSR acquisition period.

Using the Morlet wavelet transformation ${ }^{70}$ implemented in FieldTrip (http://www.fieldtriptoolbox.org/) ${ }^{71}$, we transformed ECoG voltage signals into time-frequency bins ( $2 \mathrm{~Hz}$ frequency bins; three cycles for each frequency) sliding in $1 \mathrm{~ms}$ steps. The present study excluded recording sites within $1.5 \mathrm{~cm}$ of a given stimulus pair from the analysis of inter-lobar effective connectivity ${ }^{72,73}$. We measured the percent change of CCSR 
amplitude relative to the 50-200 ms pre-stimulus baseline period. The CCSR percent change was computed for each frequency bin at 20-60 Hz range within 10-50 ms post-stimulus for early CCSRs and between $2-20 \mathrm{~Hz}$ within $50-300 \mathrm{~ms}$ post-stimulus for late CCSRs. The magnitude of the local time-frequency peak that survived a cluster-based permutation test $(n=200$; cluster size threshold $a=0.05)$ was considered the strength of significant effective connectivity from the stimulus toward the recording electrode site (Fig. $\mathbf{~}^{74}$. When an electrode site was stimulated multiple times with different pairs, we computed the average connectivity from a given stimulus site (Fig. 1B).

\section{Determination of factors predictive of the strength ofdirectinter-lobar effective connectivity}

Prediction of direct effective connectivity strength between temporal and extratemporal lobe sites was accomplished using a set of mixed model analyses (Figs. 5a-b). Mixed models were conducted using the Matlab Statistics Toolbox (The MathWorks, Inc., Natick, MA, USA), and the significance was set at a false discovery rate (FDR)-corrected $p$-value of 0.05 . The dependent variable in each mixed model was the percent change of early CCSR-based effective connectivity in a given direction. The fixed-effect predictor variables included: [a] the interaction of highgamma activity (i.e., multiplication of percent change) during a given PC stage at the stimulus and recording sites, [b] the Euclidean distance $(\mathrm{mm})$ from the stimulus site to each recording site, [c] patient age (years), [d] the hemisphere of electrode placement (left/right), [e] severity of epilepsy-related burden, estimated by the number of oral antiepileptic drugs (AEDs) immediately before the electrode implantation ${ }^{58,75}$, [f] naming speed, assessed by the median response time during the auditory naming task (seconds), [g] the lobe in which the SOZ was identified (i.e., frontal, temporal or parietal/occipital lobe), and [h] the presence of an MRI-visible cortical lesion ${ }^{58}$. Intercept and patient were treated as random factors.

Two sets of ancillary mixed model analyses were conducted to assess the effect of language proficiency-adjusted age and to predict the strength of indirect effective connectivity. Twenty-five patients underwent the Clinical Evaluation of Language Fundamentals-Fourth Edition (CELF-4) before the ECoG recording ${ }^{76}$. The mixed model to assess the effect of language proficiency-adjusted age on the strength of CCSRbased direct effective connectivity was analogous to those described above but incorporated 'the square root of [patient age $\times$ standardized CELF-4 language score]' instead of 'patient age.' The second set of ancillary mixed models to predict the strength of late CCSR (Fig. 4) were analogous to the above but replaced early CCSR with late CCSR percent change as the dependent variable (Figs. 5c-d).

\section{D tractography visualization of rapid neural propagations}

Using DWI data acquired from 27 patients, we created animations to visualize, at the group level, the spatial trajectory of stimulation-induced neuronal activity along with corresponding high-gamma interaction values (Videos S1-S2). The basic dynamic tractography methodology has been reported previously ${ }^{49}$. Briefly, before the subdural electrode placement, diffusion-weighted images were collected on a 3T GE Signa MRI scanner, with a multislice single-shot diffusion-weighted echo-planar imaging sequence at $T R=12,500 \mathrm{~ms}, T E=88.7 \mathrm{~ms}, \mathrm{FOV}=24 \mathrm{~cm}, 128 \times 128$ acquisition matrix, contiguous $3 \mathrm{~mm}$ thickness axial slices to cover the entire brain using 55 isotropic gradient directions with $b=1000 \mathrm{~s} / \mathrm{mm}^{2}$, number of excitations $=1$, and single $b=0$ image ${ }^{56}$. We estimated anatomically-constrained probabilistic DWI tractography ${ }^{77,78}$, at the wholebrain level, using the fiber orientation distributions (FOD) image via the iFOD2 algorithm ${ }^{79}$, a seed density of 6000 seeds/voxel, minimum/maximum streamline length of $20 / 250 \mathrm{~mm}$, and a maximum angle of $70^{\circ}$. Electrode coordinates were first moved from the $\mathrm{T} 1$ to the DWI space using an affine transformation derived from the DWI b0 images and T1 images ${ }^{80}$, then translated from the grey matter surface to the nearest white matter coordinate using FreeSurfer algorithms. We placed a $4 \mathrm{~mm}$ radius sphere around each DWI-space electrode coordinate to extract electrode pair-specific streamlines. To estimate the velocity of early CCSRs, we divided the minimum streamline length of each electrodepair streamline bundle by the local peak latency of the corresponding early CCSR. The resulting movies visualized early CCSR-based neural propagations' dynamics and strengths along DWI-based streamlines between cortical sites engaged during each PC-defined linguistic processing stage.

Using open-source DWI data collected from the 1,065 healthy participants in the Human Connectome Project (referred to as HCP1065; http://brain.labsolver.org/diffusion-mri-templates/hcp-842-hcp-1021 ${ }^{81}$ ), we likewise determined the dynamics, strengths, and trajectories of early CCSR-induced neural propagations as computed with data from all 37 patients. We previously reported the methodological details of the usage of open-source DWI data ${ }^{50}$. In short, we placed a $4 \mathrm{~mm}$ radius sphere around each standardized Montreal Neurological Institute (MNI)space electrode coordinate to extract electrode pair-specific streamlines. Significant streamlines were estimated with the following criteria: a fractional anisotropy threshold of 0.5 , minimum/maximum streamline length of 20/250 mm, a maximum angle of $70^{\circ}$, and step size of $0.3 \mathrm{~mm}$. We used a Pearson correlation test to determine how well the velocities computed with individual DWI data corresponded to those calculated with open-source DWI data.

We quantified the proportion of each anatomical pathway that supported observed direct inter-lobar effective connectivity between given PCAbased linguistic processing stages, using the Clopper-Pearson method ${ }^{82}$. We used all 37 patients' CCSR data and open-source HCP1065 DWI data for this analysis. All streamlines satisfying the following criteria were included for this analysis: [a] naming-related high-gamma activity was augmented at temporal and extratemporal lobe sites during specific PCA-based linguistic processing stages (Fig. 3), and [b] a given streamline was associated with a significant local peak of early CCSR (Fig. 4). Each included streamline was classified into one of the following 
anatomical pathways of interest: [1] arcuate fasciculus, [2] cingulum, [3] extreme capsule, [4] frontal aslant tract, [5] inferior fronto-occipital fasciculus, [6] inferior longitudinal fasciculus, [7] middle longitudinal fasciculus, [8] parietal aslant tract, [9] superior longitudinal fasciculus, [10] uncinate fasciculus, and [11] vertical occipital fasciculus (http://www.bic.mni.mcgill.ca/ServicesAtlases/ICBM152NLin200983,84).

\section{Data and code availability}

All data used in the present study are available upon request to the corresponding author. We plan to provide our MATLAB codes in GitHub.com following the publication. We are pleased to re-analyze the data and give the results based on specific suggestions from readers to improve our understanding of the neurobiology of language.

\section{Results}

\section{Linguistic processing stages based on the principal component analysis (PCA)}

The first three PCs explained approximately $95 \%$ of the variance in naming-related high-gamma modulation patterns (Fig. $3 \mathbf{b}$ ); thus, the following analyses focus on the effective connectivity between these PCA-based language sites. As shown in the PC coefficient matrix (Figs. 3a and c), PC1 was primarily attributed to high-gamma augmentation elicited during overt speech-response and self-monitoring one's own voice; electrode sites showing the PC1 high-gamma pattern were distributed bilaterally in the Rolandic gyri as well as STG (Fig. 3d). PC2 was attributed to high-gamma augmentation elicited during a sentence stimulus listening period (Figs. 3a and c); PC2 sites were distributed mainly in the STG and posterior MTG (Fig. 3e). PC3 was attributed to high-gamma augmentation elicited mainly between sentence stimulus offset and response onset (Figs. 3a and c); PC3 sites were distributed predominantly in the left hemisphere, including widespread posterior temporal and frontal neocortical regions (Fig. 3f).

\section{Factors predictive ofdirectinter-lobar effective connectivity}

The mixed model analyses confirmed the hypothesis that the magnitude of naming-related co-activation predicts the strength of direct interlobar effective connectivity (i.e., the magnitude of early CCSR local peak). Across all six (PCs 1-3, and two directions) mixed models, the interaction of naming-related HGA was a significant predictor of the strength of direct effective connectivity (Figs. 5a-b; Supplementary Tables

S1-S6). For example, an increased interaction of temporal and extratemporal lobe naming-related HGA during the PC3 language stage predicted an increased strength of direct inter-lobar effective connectivity from PC3 temporal lobe language sites (mixed model estimate: +106.1\%; FDRcorrected p-value: <0.001; t-value: 5.3; DF: 7697; Fig. 5a and Supplementary Table S3).

Patient age was likewise found to be a key predictor of the strength of direct effective connectivity. Positive associations between age and the connectivity strength were observed in the extratemporal-to-temporal direction across all three PC networks and bidirectionally in PC3. For example, each one-year increase in patient age likewise predicted an increased strength of direct inter-lobar effective connectivity from PC3 temporal lobe language sites (mixed model estimate: +1.2\%/year; FDR-corrected p-value: <0.001; t-value: 4.9; DF: 7697; Fig. 5a and Supplementary Table S3). However, when language proficiency-adjusted age was used, the relationship to the strength of direct effective connectivity was not replicated (See the details in Supplementary Tables S7-S9). The age effect on the effective connectivity in the opposite direction was significant (Fig. 5b) regardless of the types of underlying PC language sites (Supplementary Tables S4-S6).

The aforementioned significant effects were all independent of those of inter-electrode Euclidean distance as well as epilepsy-related factors. For example, longer Euclidean distance between stimulus and recording sites was associated with a decreased strength of direct effective connectivity across all PC-defined language networks. The number of AEDs was found to negatively predict the connectivity strength in the temporal-to-extratemporal direction across all three PC networks (Figs. 5a-b; Supplementary Tables S1-S6).

\section{Factors predictive ofindirectinter-lobar effective connectivity}

The mixed model analyses also confirmed the positive relationship between the magnitude of naming-related HGA interaction and the strength of indirect inter-lobar effective connectivity (i.e., the magnitude of late CCSR local peak) from the temporal to the extratemporal lobe sites in all three PC-defined language networks (Fig. 5c). The high-gamma interaction effect, however, was significant only for PC1 in the extratemporal-totemporal direction (Figs. 5c-d; Supplementary Tables S10-S13). In contrast to direct effective connectivity which did not show any hemispheric differences, a bias towards the left hemisphere was observed for indirect effective connectivity projecting from all PC-define language sites in the temporal lobe (mixed model estimate: 18.0-32.5\%; Fig. 5c; Supplementary Tables S10-S12). Older age was associated with an increased strength of indirect effective connectivity from all extratemporal lobe PC-defined language sites (Fig. 5d; Supplementary Tables S13-15). Similar to direct effective connectivity, decreased inter-electrode Euclidean distance likewise predicted the strength of indirect effective connectivity (Figs. 5c-d).

\section{Direct inter-lobar effective connectivity across sites supporting the same and different linguistic processing stages}




\section{D dynamic tractography}

Videos $\mathbf{S} 1$ and $\mathbf{S} 2$ best demonstrate the behavioral mode of early CCSR-based neural propagations across PC-defined language stages. In these videos, we localized streamline estimates of white matter pathways directly connecting regions supporting PC-defined linguistic stages using DWI data acquired from 27 patients. Evidence of CCSR connections and DWI connection provided evidence of a physiological relationship between stimulus and recording sites. Readers will find that red streamlines are associated with large moving dots. The video snapshots (Fig. 7) present the anatomical clustering of 'collaborative' (high-gamma co-augmentation at the stimulus and the recording sites; red streamline) and 'uncollaborative' (high-gamma augmentation at the stimulus site but high-gamma attenuation at the recording endpoint; blue streamlines) relationships at the group level in template space. For example, the PC1 temporal lobe language sites collaborated mainly with the precentral gyrus bilaterally (Fig. 7a), whereas the PC3 temporal lobe language sites collaborated with widespread regions in the left hemisphere (Fig. 7c).

The additional analysis using open-source DWI scans of healthy individuals and CCSR-based effective connectivity derived from all 37 study patients provided generalizable language-network models (Supplementary Fig. S2). Before using the DWI data to analyze the anatomical distribution of inter-lobar effective connectivity pathways between language sites, we first verified that, where the open-source DWI data and the individual data overlapped, they had similar relationships to the CCSR data. Specifically, we found that the velocity of CCSR-based neural propagations was highly correlated between individual and open-source DWI data $(r=0.81, \mathrm{p}$-value $<0.001$ in the 338 commonly available streamlines in the temporal-to-extratemporal direction; $r=0.80$; $p$-value $<0.001$ in the 189 streamlines in the extratemporal-to-temporal direction). Assessment of all 37 patients' CCSR data using the open-source DWI data indicated that the arcuate fasciculus allowed approximately two-thirds of the temporal-to-extratemporal lobe neural propagations; the uncinate fasciculus was used for less than $2 \%$ of such propagations (Table 2and Supplementary Fig. S2). Conversely, the arcuate fasciculus was used for up to half of the extratemporal-to-temporal lobe neural propagations; the uncinate fasciculus was used for up to $6 \%$ of such propagations (Table 2and Supplementary Fig. S2). 
Table 2

Composition proportion of naming-related pathways from and to the temporal lobe based on the open-source DWI data.

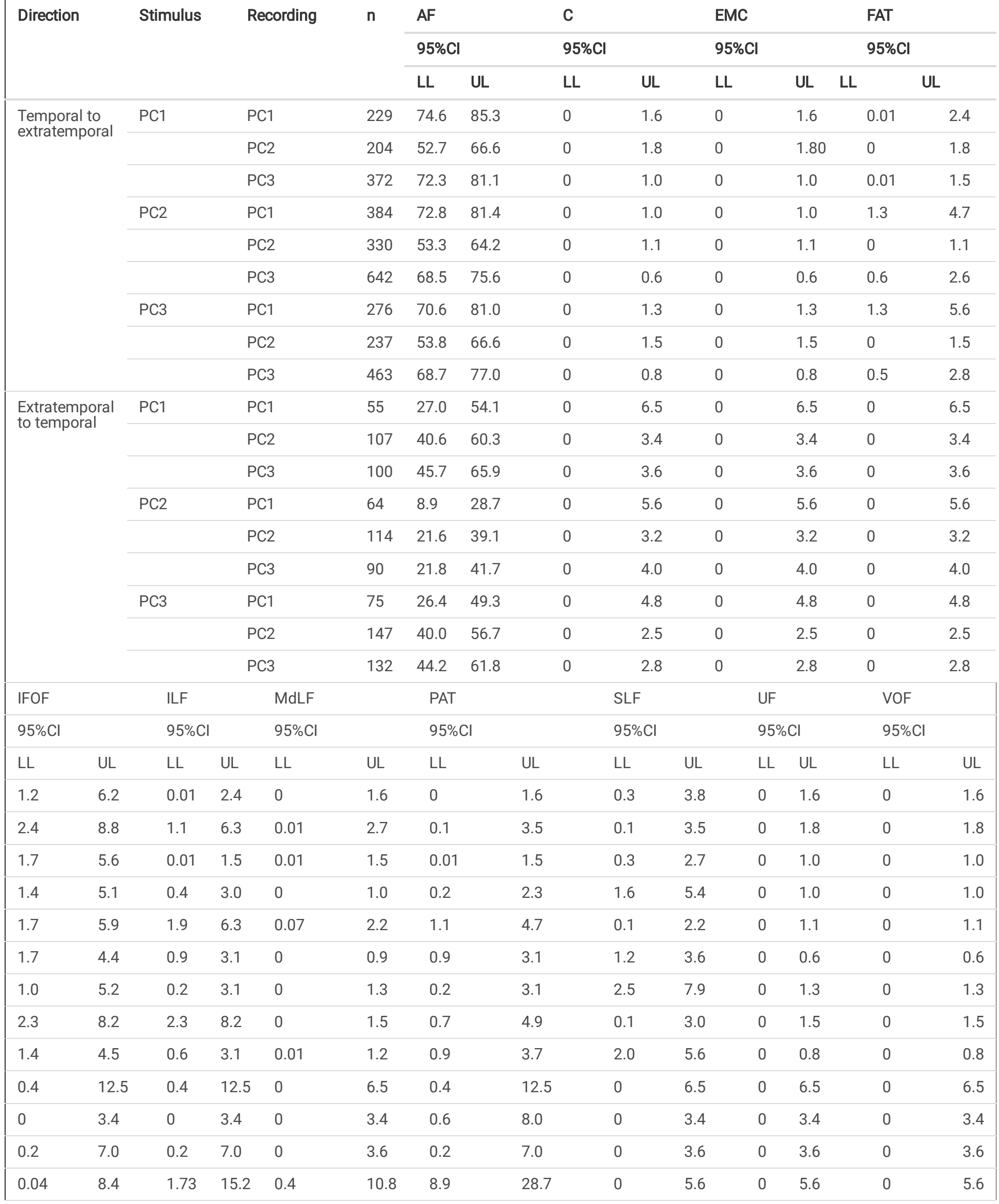




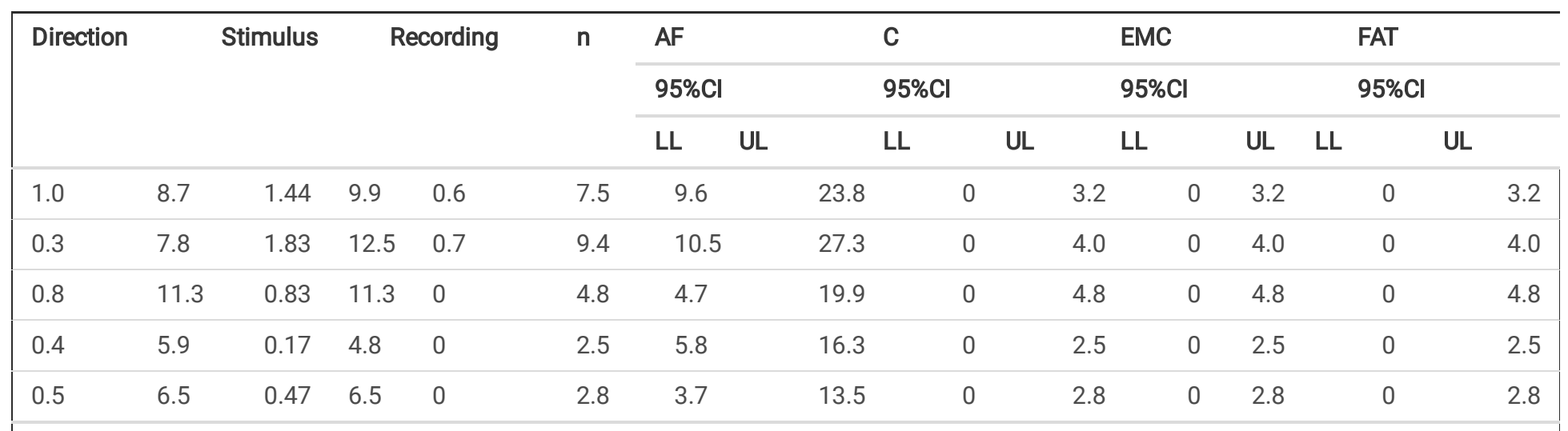

\section{Discussion}

\section{The novelty of the present study}

Our multimodality study quantitatively determined the strength, dynamics, and direction, and anatomical distribution of inter-lobar effective connectivity between areas supporting the same and different linguistic stages in the developing brain. Our novel 6D tractography animations visualized the trajectory of inter-lobar CCSR-based neural propagations from specific language-related sites, along with correlated metrics, at the whole-brain level. Extratemporal lobe sites that support semantic retrieval had direct effective connectivity preferentially from and toward temporal lobe sites supporting the same linguistic stage. Conversely, extratemporal lobe sites activated during overt responses had direct effective connectivity with temporal lobe language sites regardless of supporting linguistic stages. Age-dependent strengthening of temporal-toextratemporal lobe direct effective connectivity preferentially took place between language areas supporting semantic retrieval. The arcuate fasciculus consisted of about two-thirds of the auditory language-related effective connectivity pathways in the temporal-to-extratemporal direction but only up to half of those in the opposite direction. The uncinate fasciculus consisted of only less than $2 \%$ of those in the temporalto-extratemporal direction and up to $6 \%$ of those in the opposite direction.

\section{PC-based categorization of linguistic processing stages}

Cortical regions classified as PC1 were characterized by neural activation during the 300-ms period after the response onset (Fig. 3). The anatomical distribution of PC1 sites was mainly in the bilateral Rolandic gyri and STG, which are suggested to be an integral part of the 'phonological loop'7,8,12,19-23. High-frequency electrical stimulation of cortical areas in these regions has been shown to result in speech arrest, face motor symptoms, or auditory hallucination ${ }^{5}$. Therefore we interpreted the PC1 network to be highly engaged in supporting motor output and interpreting auditory perceptions, in particular the monitoring of one's own vocal sounds.

PC2 was characterized by neural activation mainly during the sentence listening period (Fig. 3c) and was distributed mostly in the bilateral STG and posterior MTG (Fig. 3e). High-frequency stimulation of these cortical areas has been shown to elicit auditory hallucination, impaired syllable discrimination, or receptive aphasia ${ }^{5,85}$. Prior ECoG, fMRI, and lesion-deficit studies have suggested that these anatomical regions map acoustic information onto phonological representations ${ }^{86-89}$. We thus interpret PC2 sites as engaged in support of perceptual processing of auditory sentence stimuli. It is also plausible that the PC2 high-gamma pattern reflects some of the neural engagement required for semantic understanding of each word. In contrast, some investigators suggest that much of the higher-order processing to understand the entire semantic context occurs, mainly after a full sentence is heard ${ }^{90,91}$.

PC3 was characterized by neural activation between sentence offset and response onset (Fig. 3c). The PC3 sites were distributed predominantly in the left hemisphere, including widespread posterior temporal and frontal neocortical regions (Fig. 3f). We previously reported that ESM at these regions frequently elicited expressive aphasia characterized by the inability to generate a relevant answer on time while the patient could repeat heard sounds and recall the heard sentence ${ }^{5}$. Therefore, PC3 sites are believed to support the retrieval of semantically coherent words.

\section{Proposed model of the inter-lobar network dynamics supporting auditory naming}

As best presented in Videos S1-S2, the temporal lobe language areas have developed effective connectivity pathways allowing rapid sharing of phonologic and semantic representations with the extratemporal lobe structures supporting the same and different linguistic stages.

Approximately two-thirds of such temporal-to-extratemporal effective connectivity lies in the arcuate fasciculus, whereas less than $2 \%$ in the uncinate (Figs. 7a-c and Table 2). Conversely, the arcuate and uncinate fasciculi support up to $50 \%$ and $6 \%$ of the effective connectivity in the opposite direction (Figs. 7d-f and Table 2). 
Our multimodal study has expanded the existing models suggesting that the arcuate fasciculus consists of a central pathway allowing temporal-to-extratemporal communications essential for auditory naming 3,4,10,11. The novel aspects of our study include the quantification of the functional specificity and direction dependency of the underlying white matter fasciculi. We propose that extratemporal lobe regions supporting semantic retrieval have direct effective connectivity from widespread temporal lobe language regions but twice more preferentially from those supporting the same linguistic stage (Fig. 6c). Conversely, extratemporal lobe regions consisting of the 'phonological loop' have effective connectivity uniformly from the temporal lobe language regions. Our results support the models suggesting that much of the language-related neural propagations from the temporal lobe occur directly through the arcuate fasciculus ${ }^{4,11}$. In contrast, we suggest that neural propagations toward the temporal lobe originate from more diffuse extratemporal regions, including those outside the arcuate fasciculus territory such as the anterior IFG, parietal, and occipital regions (Table 2). Verbal discourse is suggested to require self-assessments and monitoring of voice pitch, semantic coherence of answers, and contextual appropriateness ${ }^{7,8,92,93}$. Further studies are warranted to determine if the extratemporal-to-temporal effective connectivity outside the arcuate fasciculus in each hemisphere would be involved in the audio-visual integration supporting the pragmatic aspect of language ${ }^{94,95}$.

Our results do not support a central role for temporal-to-extratemporal connectivity along the uncinate fasciculus in auditory naming ${ }^{9}$. Previous studies have likewise reported unimpaired language function during high-frequency stimulation of the uncinate fasciculus ${ }^{96}$ and after left anterior temporal lobectomy ${ }^{97}$. Our auditory naming-related network dynamics reported in the present study should not be generalized to those enabling visual language. It remains unknown how much the uncinate fasciculus would contribute to inter-lobar effective connectivity between visual language areas. Indeed, our previous ECoG study reported that a picture naming task, compared to an auditory naming task, elicited more intense high-gamma augmentation in the anterior fusiform and entorhinal cortices ${ }^{62}$. These ventral temporal lobe structures are anatomically connected to the anterior IFG via the uncinate fasciculus ${ }^{98}$.

Our sturdy by no means disproves the presence of indirect connectivity pathways between temporal and extratemporal lobe sites. Indeed, we observed early CCSRs between the STG and inferior parietal lobe, which might contribute to indirect information transfer between the STG and the frontal lobe 2,48 .

\section{Proposed model of the inter-lobar connectivity development after age five}

Our study proposes that age-dependent strengthening of inter-lobar effective connectivity after age five preferentially occurs between language areas supporting semantic retrieval. The present study demonstrated that older age increased the strength of direct temporal-to-extratemporal lobe effective connectivity preferentially between sites supporting semantic retrieval processing (Fig. 5a and Supplementary Table S3).

It is plausible to hypothesize that the numerous transfers of the neural representations of semantic information, which occurs over the lifespan ${ }^{99}$, may strengthen inter-lobar effective connectivity via white matter pathways. Verbal conversation is a face-to-face communication style typical across the lifespan. Children and young adults are estimated to exchange up to several thousands of spoken sentences a day ${ }^{100}$, implying heavy utilization of the white matter networks between the temporal and extratemporal lobe structures. A putative mechanism for the age-dependent increases in connectivity may be a strengthening of myelination. Studies of cultured cells have demonstrated that increased action potential firing strengthens myelination ${ }^{101-103}$. There is likewise evidence for use-dependent changes in white matter pathways. A DWI study of 8- to 12-year-old readers reported that a six-month training including 100 hours of intensive remedial reading instruction additively increased the fractional anisotropy (a measure of underlying myelination) of the left-hemispheric cortico-cortical white matter tracts ${ }^{104}$. Enormous changes in inter-lobar effective connectivity are expected to occur during infancy and toddlerhood ${ }^{9}$; thus, further studies including younger children are warranted to determine if the temporal-to-extratemporal lobe connectivity supporting the phonological process are strengthened earlier than that supporting the semantic process.

\section{Hemispheric asymmetry ofindirecteffective connectivity from the temporal lobe}

One of our novel findings was that the left hemisphere, compared to the right, had increased strength of late CCSR-based effective connectivity from temporal lobe language sites (Fig. 5c and Supplementary Tables S10-S12). Late CCSRs, characterized by augmentation of low-frequency activity at $4-7 \mathrm{~Hz}$, are suggested to indicate post-excitatory neural inhibition ${ }^{39,41}$, as reflected by reduced firing in simultaneous single-neuron recording ${ }^{44,46}$. Likewise, in the current study, the average frequency of the significant late CCSR local peaks in temporal-to-extratemporal lobe direction was $6.8 \mathrm{~Hz}$, indicating inhibitory-functioning connectivity. The observation of left-hemispheric bias in late CCSRs raises the possibility that the left hemisphere may have more robust inhibitory networks governing inter-lobar effective connectivity.

It is possible that the left-hemispheric dominant late CCSRs, which may reflect inhibitory activity, account for the left-hemispheric dominant naming-related high-gamma augmentation during the semantic retrieval period (Fig. 3f) ${ }^{5,29}$. Task-related high-gamma augmentation has been suggested to be attributed to the firing of inhibitory interneurons at high-gamma frequencies ${ }^{105-110}$. Some have reported that increased GABA 
level measured by magnetic resonance spectroscopy (MRS) predicted a high peak frequency and power of task-induced gamma (< $70 \mathrm{~Hz}$ ) activity measured by magnetoencephalography ${ }^{111,112}$, whereas others failed to replicate such findings ${ }^{113}$.

\section{Methodological considerations}

While task-based functional connectivity, CCSR-based effective connectivity, and DWI-based structural connectivity have specific strengths, each method has an inevitable limitation. Previous studies using Granger causality reported that cortical sites showing task-related highgamma augmentation during a shared time window could propagate neural activity between them ${ }^{12,13,20,114}$. However, Granger causality cannot consistently distinguish direct versus indirect connectivity. Early CCSRs are a valuable measure to quantify the strength of long-range direct effective connectivity 39,41 , but do not reveal the anatomical white matter structure which supports the observed connectivity. Conversely, DWI-based tractography is a powerful tool to characterize anatomical white matter networks between two regions ${ }^{11}$, but does not measure the directionality or function of the pathway. Our 6D dynamic tractography approach integrates all three modalities into a single model and visualization, which revealed the white matter pathways supporting rapid neural propagations between sites engaged to specific linguistic stages. We hypothesize that the dynamic trajectories observable via 6D tractography are more likely to be used for auditory naming if the remote areas connected are simultaneously engaged in the same linguistic stage $\mathrm{e}^{12,13,20,114}$. Indeed, we found that early CCSRs were larger when the stimulus and recording sites showed high-gamma augmentation during the same linguistic stage. However, it is possible that although a direct connection exists (as evidenced by a CCSR), this is not the pathway being utilized during the task. One cannot blindly presume that all of the dynamic trajectories on the 6D tractography (particularly those via blue streamlines; Fig. 7) are active during auditory naming. Single-pulse electrical stimulation forces a cortical site to induce neural propagations, some of which may or may not take place during a cognitive task.

We believe that the risk of our late CCSRs being contaminated by stimulation-induced pathological/epileptogenic high-frequency oscillations (HFOs) is small ${ }^{115}$ because we excluded the SOZ as well as areas affected by interictal spikes or structural lesions from the CCSR analysis. Furthermore, the mean latencies of our late CCSRs from temporal lobe sites were $<120$ ms, whereas stimulation-induced pathological HFOs typically occur approximately $250 \mathrm{~ms}$ and after $^{115}$. TMS-based single-pulse stimulation at a central region in healthy adults was reported to elicit low-frequency responses in remote areas at a latency of roughly $100 \mathrm{~ms}^{116}$.

A benefit but also a limitation of 6D tractography is that it leverages the intersection of data from CCSR and DWI-based tractography. The use of early CCSRs to constrain tractography helps minimize the influence of false positives in tractography results ${ }^{49}$. However, the low resolution and signal-to-noise ratio of tractography may mean that false negatives are potentially present as well. Therefore, while we can have confidence in the positive results observed in the $6 \mathrm{D}$ dynamic tractography movies, they likely represent a subset of the full network. The velocity of neural propagations was highly correlated between the datasets derived from individual and open-source DWI (Pearson $r=0.8)$. This observation indicates that the anatomical trajectories of streamlines supporting observed effective connectivity were spatially similar between epilepsy patients and healthy participants in the human connectome project (http://brain.labsolver.org/diffusion-mri-templates/hcp-842-hcp-1021 ${ }^{81}$ ).

Our mixed model analysis effectively controlled for the effect of epilepsy-related factors on CCSR and DWI measures. The reported effects of linguistic processing stages and patient age on the strength of inter-lobar effective connectivity were all independent of epilepsy-related factors as well as the Euclidean distance between stimulus and recording sites. For example, an increased number of oral antiepileptic drugs at the time of surgery was associated with reduced strength of direct effective connectivity from the temporal lobe sites supporting the semantic process (Fig. 5a and Supplementary Table S3). Either antiepileptic drugs or severe epilepsy requiring polytherapy may account for this observation. A previous study of healthy adults reported that sodium-channel blockers but not GABA-mediated antiepileptic drugs elevated the motor threshold on transcranial magnetic stimulation-based mapping ${ }^{117}$. In contrast, sodium-channel blocker monotherapy did not alter the amplitude of short-latency somatosensory evoked potentials ${ }^{118}$. Second, a larger number of oral antiepileptic drugs generally reflect greater severity of seizure burden, including more severe cognitive dysfunction ${ }^{75}$. Several fMRI studies have reported that resting-state connectivity is altered severely within and slightly outside the SOZ ${ }^{119-121}$.

Because the extent of electrode placement was guided purely by clinical needs, most patients underwent unilateral electrode placement. As shown in Fig. 1, the spatial extent of ECoG sampling across patients was grossly symmetric. The present study was not designed to clarify the role of interhemispheric connectivity in auditory naming. Our recent stereo-EEG study of three patients reported that both temporal and frontal lobe neocortices had direct effective connectivity to the homotopic areas via either the corpus callosum or anterior commissure and that the latency of early CCSR was less than $50 \mathrm{~ms}^{50}$.

The present study focused on assessing large-scale, inter-lobar connectivity networks from and toward the temporal lobe on each hemisphere. We indeed excluded recording sites within $1.5 \mathrm{~cm}$ from a given stimulus pair from the analysis ${ }^{72,73}$. Thus, at this moment, we cannot propose a model of short-range network dynamics supporting auditory naming. Employment of smaller intensity of single-pulse stimulation, an ECoG amplifier with a higher sampling rate, and increased density of intracranial electrode arrays may allow us to quantify the short-range effective connectivity network. 


\section{Abbreviations}

AEDs = antiepileptic drugs. BOLD = blood-oxygen-level-dependent. CCEPs = cortico-cortical evoked potentials. CCSRs $=$ cortico-cortical spectral responses. $\mathrm{DWI}=$ diffusion weighted imaging. $\mathrm{ECOG}=$ electrocorticography. $\mathrm{ESM}=$ electrical stimulation mapping. $\mathrm{FOD}=$ fiber orientation distributions. HFOs = high-frequency oscillations. IFG = inferior frontal gyrus. ITG = inferior temporal gyrus. iEEG = intracranial electroencephalography. IQR = interquartile range. $\mathrm{MFG}=$ middle frontal gyrus. $\mathrm{MTG}=$ middle temporal gyrus. SD = standard deviation. $\mathrm{SE}=$ standard error. SOZ = seizure onset zone. STG = superior temporal gyrus. TMS = transcranial magnetic stimulation. $\mathrm{MNI}=\mathrm{Montreal}$ Neurological Institute.

\section{Declarations}

\section{Acknowledgements}

We are grateful to Alanna Carlson, MS, LLP, Karin Halsey, BS, REEGT., Jamie MacDougall, RN, BSN, CPN at Children's Hospital of Michigan for the collaboration and assistance in performing the studies described above.

\section{Author contributions}

M.S., B.H.S., and E.A. made the conception and the design of this work.

R.R., A.F.L., S.S., and E.A. performed data acquisition.

M.S., B.H.S., J.W.J., A.S., Y.N., and E.A. analyzed data and prepared all figures.

M.S., B.H.S., and E.A. interpreted results.

M.S., B.H.S., and E.A. wrote the main manuscript text.

All authors critically reviewed and revised the manuscript.

\section{Competing interests}

The authors have no conflicts of interest to report. We confirm that we have read the Journal's position on issues involved in ethical publication and affirm that this report is consistent with those guidelines.

\section{Funding}

This work was supported by NIH grants NS064033 (to E.A.) and NS089659 (to J.W.J.).

\section{References}

1. Hamberger, M. J., Seidel, W. T., Mckhann, G. M., Perrine, K. \& Goodman, R. R. Brain stimulation reveals critical auditory naming cortex. Brain $128,2742-2749$ (2005).

2. Hickok, G. \& Poeppel, D. The cortical organization of speech processing. Nat Rev Neurosci 8, 393-402 (2007).

3. Rauschecker, J. P. \& Scott, S. K. Maps and streams in the auditory cortex: nonhuman primates illuminate human speech processing. Nat Neurosci 12, 718-724 (2009).

4. Chang, E. F., Raygor, K. P. \& Berger, M. S. Contemporary model of language organization: an overview for neurosurgeons. J Neurosurg 122, 250-261 (2015).

5. Nakai, Y. et al. Three- and four-dimensional mapping of speech and language in patients with epilepsy. Brain 140, 1351-1370 (2017).

6. Towle, V. L. et al. ECoG gamma activity during a language task: differentiating expressive and receptive speech areas. Brain 131, 20132027 (2008).

7. Pei, X. et al. Spatiotemporal dynamics of electrocorticographic high gamma activity during overt and covert word repetition. Neuroimage 54, 2960-2972 (2011).

8. Greenlee, J. D. W. et al. Sensory-Motor Interactions for Vocal Pitch Monitoring in Non-Primary Human Auditory Cortex. Plos One 8, e60783 (2013).

9. Skeide, M. A. \& Friederici, A. D. The ontogeny of the cortical language network. Nat Rev Neurosci 17, 323-332 (2016).

10. Hagoort, P. The neurobiology of language beyond single-word processing. Science 366, 55-58 (2019). 
11. Catani, M. \& Mesulam, M. The arcuate fasciculus and the disconnection theme in language and aphasia: History and current state. Cortex 44, 953-961 (2008).

12. Flinker, A. et al. Redefining the role of Broca's area in speech. Proc National Acad Sci 112, 2871-2875 (2015).

13. Collard, M. J. et al. Cortical subnetwork dynamics during human language tasks. Neuroimage 135, 261-272 (2016).

14. Kambara, T. et al. Spatio-temporal dynamics of working memory maintenance and scanning of verbal information. Clin Neurophysio/ 128, 882-891 (2017).

15. Trimmel, K. et al. Left temporal lobe language network connectivity in temporal lobe epilepsy. Brain 141, 2406-2418 (2018).

16. Simmonds, D. J., Hallquist, M. N., Asato, M. \& Luna, B. Developmental stages and sex differences of white matter and behavioral development through adolescence: A longitudinal diffusion tensor imaging (DTI) study. Neuroimage 92, 356-368 (2014).

17. Blakemore, S.-J. Imaging brain development: The adolescent brain. Neuroimage 61, 397-406 (2012).

18. Geschwind, N. Disconnexion Syndromes in Animals and Man: Part I. Neuropsychol Rev 20, 128-157 (2010).

19. Cogan, G. B. et al. Sensory-motor transformations for speech occur bilaterally. Nature 507, $94-98$ (2014).

20. Nishida, M. et al. Brain network dynamics in the human articulatory loop. Clin Neurophysiol 128, 1473-1487 (2017).

21. Schubotz, R. I., Cramon, D. Y. von \& Lohmann, G. Auditory what, where, and when: a sensory somatotopy in lateral premotor cortex. Neuroimage 20, 173-185 (2003).

22. Bangert, M. et al. Shared networks for auditory and motor processing in professional pianists: Evidence from fMRI conjunction. Neuroimage 30, 917-926 (2006).

23. Pulvermüller, F. et al. Motor cortex maps articulatory features of speech sounds. Proc National Acad Sci 103, 7865-7870 (2006).

24. Ojemann, G., Ojemann, J., Lettich, E. \& Berger, M. Cortical language localization in left, dominant hemisphere: An electrical stimulation mapping investigation in 117 patients. J Neurosurg 71, 316-326 (1989).

25. Springer, J. A. et al. Language dominance in neurologically normal and epilepsy subjectsA functional MRI study. Brain 122, 2033-2046 (1999).

26. McDonald, C. R. et al. Multimodal imaging of repetition priming: Using fMRI, MEG, and intracranial EEG to reveal spatiotemporal profiles of word processing. Neuroimage 53, 707-717 (2010).

27. Schoffelen, J.-M. et al. A 204-subject multimodal neuroimaging dataset to study language processing. Sci Data 6, 17 (2019).

28. Leuthardt, E. C. et al. Temporal evolution of gamma activity in human cortex during an overt and covert word repetition task. Front Hum Neurosci 6, 99 (2012).

29. Ikegaya, N. et al. Spatiotemporal dynamics of auditory and picture naming-related high-gamma modulations: a study of Japanesespeaking patients. Clin Neurophysiol 130, 1446-1454 (2019).

30. Lachaux, J.-P., Axmacher, N., Mormann, F., Halgren, E. \& Crone, N. E. High-frequency neural activity and human cognition: Past, present and possible future of intracranial EEG research. Prog Neurobio/ 98, 279-301 (2012).

31. Jacobs, J., Kahana, M. J., Ekstrom, A. D. \& Fried, I. Brain Oscillations Control Timing of Single-Neuron Activity in Humans. J Neurosci 27, 3839-3844 (2007).

32. Whittingstall, K. \& Logothetis, N. K. Frequency-Band Coupling in Surface EEG Reflects Spiking Activity in Monkey Visual Cortex. Neuron 64, 281-289 (2009).

33. Manning, J. R., Jacobs, J., Fried, I. \& Kahana, M. J. Broadband Shifts in Local Field Potential Power Spectra Are Correlated with SingleNeuron Spiking in Humans. J Neurosci 29, 13613-13620 (2009).

34. Nishida, M., Juhász, C., Sood, S., Chugani, H. T. \& Asano, E. Cortical glucose metabolism positively correlates with gamma-oscillations in nonlesional focal epilepsy. Neuroimage 42, 1275-1284 (2008).

35. Ray, S., Crone, N. E., Niebur, E., Franaszczuk, P. J. \& Hsiao, S. S. Neural Correlates of High-Gamma Oscillations (60-200 Hz) in Macaque Local Field Potentials and Their Potential Implications in Electrocorticography. J Neurosci 28, 11526-11536 (2008).

36. Scheeringa, R. et al. Neuronal Dynamics Underlying High- and Low-Frequency EEG Oscillations Contribute Independently to the Human BOLD Signal. Neuron 69, 572-583 (2011).

37. Arya, R., Horn, P. S. \& Crone, N. E. ECoG high-gamma modulation versus electrical stimulation for presurgical language mapping. Epilepsy Behav 79, 26-33 (2018).

38. Kojima, K. et al. Clinical significance and developmental changes of auditory-language-related gamma activity. Clin Neurophysio/ 124, 857-869 (2013).

39. Usami, K. et al. The neural tides of sleep and consciousness revealed by single-pulse electrical brain stimulation. Sleep 42, 1-9 (2019).

40. Koubeissi, M. Z. et al. Connectivity between Perisylvian and Bilateral Basal Temporal Cortices. Cereb Cortex 22, 918 -925 (2012). 
41. Matsumoto, R., Kunieda, T. \& Nair, D. Single pulse electrical stimulation to probe functional and pathological connectivity in epilepsy. Seizure 44, 27-36 (2017).

42. Matsumoto, R. et al. Functional connectivity in the human language system: a cortico-cortical evoked potential study. Brain 127, 23162330 (2004).

43. Trebaul, L. et al. Probabilistic functional tractography of the human cortex revisited. Neuroimage 181, 414-429 (2018).

44. Alarcón, G. et al. In vivo neuronal firing patterns during human epileptiform discharges replicated by electrical stimulation. Clin Neurophysiol 123, 1736-1744 (2012).

45. Keller, C. J. et al. Mapping human brain networks with cortico-cortical evoked potentials. Philosophical Transactions Royal Soc B Biological Sci 369, 20130528 (2014).

46. Logothetis, N. K. et al. The effects of electrical microstimulation on cortical signal propagation. Nat Neurosci 13, 1283-1291 (2010).

47. Mitsuhashi, T. et al. Effects of depth electrode montage and single-pulse electrical stimulation sites on neuronal responses and effective connectivity. Clin Neurophysiol 131, 2781-2792 (2020).

48. Matsumoto, R. et al. Parieto-frontal network in humans studied by cortico-cortical evoked potential. Hum Brain Mapp 33, 2856-2872 (2012).

49. Silverstein, B. H. et al. Dynamic tractography: Integrating cortico-cortical evoked potentials and diffusion imaging. Neuroimage 215,116763 (2020).

50. Mitsuhashi, T. et al. Four-dimensional tractography animates neural propagations via distinct interhemispheric pathways. Clinical Neurophysiology. Accepted/In press (2020). Preprint at https://biorxiv.org/cgi/content/short/2020.12.11.421495v1 (2020)

51. Jolliffe, I. T. Principal component analysis. 2nd ed. (Springer-Verlag, 2002).

52. Ghosh, S. S. et al. Evaluating the validity of volume-based and surface-based brain image registration for developmental cognitive neuroscience studies in children 4 to 11years of age. Neuroimage 53, 85-93 (2010).

53. Asano, E., Juhász, C., Shah, A., Sood, S. \& Chugani, H. T. Role of subdural electrocorticography in prediction of long-term seizure outcome in epilepsy surgery. Brain 132, 1038-1047 (2009).

54. Kambara, T. et al. Presurgical language mapping using event-related high-gamma activity: The Detroit procedure. Clin Neurophysio/ 129, 145-154 (2018).

55. Stolk, A. et al. Integrated analysis of anatomical and electrophysiological human intracranial data. Nat Protoc 13, 1699-1723 (2018).

56. Jeong, J. et al. Automatic detection of primary motor areas using diffusion MRI tractography: Comparison with functional MRI and electrical stimulation mapping. Epilepsia 54, 1381-1390 (2013).

57. Pieters, T. A., Conner, C. R. \& Tandon, N. Recursive grid partitioning on a cortical surface model: an optimized technique for the localization of implanted subdural electrodes: Clinical article. J Neurosurg 118, 1086-1097 (2013).

58. Motoi, H. et al. Quantitative analysis of intracranial electrocorticography signals using the concept of statistical parametric mapping. Sci Rep-uk 9, 17385 (2019).

59. Frauscher, B. et al. Atlas of the normal intracranial electroencephalogram: neurophysiological awake activity in different cortical areas. Brain 141, 1130-1144 (2018).

60. Zijlmans, M. et al. Ictal and interictal high frequency oscillations in patients with focal epilepsy. Clin Neurophysiol 122, 664-671 (2011).

61. Uematsu, M., Matsuzaki, N., Brown, E. C., Kojima, K. \& Asano, E. Human occipital cortices differentially exert saccadic suppression: Intracranial recording in children. Neuroimage 83, 224-236 (2013).

62. Nakai, Y. et al. Four-dimensional functional cortical maps of visual and auditory language: Intracranial recording. Epilepsia 60, 255-267 (2019).

63. Papp, N. \& Ktonas, P. Critical evaluation of complex demodulation techniques for the quantification of bioelectrical activity. Biomed Sci Instrum 13, 135-145 (1977).

64. Hoechstetter, K. et al. BESA Source Coherence: A New Method to Study Cortical Oscillatory Coupling. Brain Topogr 16, 233-238 (2004).

65. Kojima, K. et al. Gamma activity modulated by picture and auditory naming tasks: Intracranial recording in patients with focal epilepsy. Clin Neurophysiol 124, 1737-1744 (2013).

66. Kojima, K. et al. Multimodality language mapping in patients with left-hemispheric language dominance on Wada test. Clin Neurophysiol 123, 1917-1924 (2012).

67. Sugiura, A. et al. Four-dimensional map of direct effective connectivity from posterior visual areas. Neuroimage 210, 116548 (2020).

68. Valentín, A. et al. Responses to single pulse electrical stimulation identify epileptogenesis in the human brain in vivo. Brain 125, 1709-1718 (2002).

69. Crowther, L. J. et al. A quantitative method for evaluating cortical responses to electrical stimulation. J Neurosci Meth 311, 67-75 (2019). 
70. Tallon-Baudry, C., Bertrand, O., Delpuech, C. \& Permier, J. Oscillatory gamma-band (30-70 Hz) activity induced by a visual search task in humans. The Journal of neuroscience: the official journal of the Society for Neuroscience 17, 722-734 (1997).

71. Oostenveld, R., Fries, P., Maris, E. \& Schoffelen, J.-M. FieldTrip: Open source software for advanced analysis of MEG, EEG, and invasive electrophysiological data. Computational intelligence and neuroscience 2011, 156869 (2011).

72. Swann, N. C. et al. Roles for the pre-supplementary motor area and the right inferior frontal gyrus in stopping action: Electrophysiological responses and functional and structural connectivity. Neuroimage 59, 2860-2870 (2012).

73. Prime, D., Woolfe, M., O’Keefe, S., Rowlands, D. \& Dionisio, S. Quantifying volume conducted potential using stimulation artefact in corticocortical evoked potentials. J Neurosci Meth 337, 108639 (2020).

74. Maris, E. \& Oostenveld, R. Nonparametric statistical testing of EEG-and MEG-data. J Neurosci Meth 164, 177-190 (2007).

75. Kwan, P. \& Brodie, M. J. Neuropsychological effects of epilepsy and antiepileptic drugs. Lancet 357, 216-222 (2001).

76. Paslawski, T. The Clinical Evaluation of Language Fundamentals, Fourth Edition (CELF-4). Can J Sch Psychology 20, 129-134 (2005).

77. Patenaude, B., Smith, S. M., Kennedy, D. N. \& Jenkinson, M. A Bayesian model of shape and appearance for subcortical brain segmentation. Neuroimage 56, 907-922 (2011).

78. Zhang, Y., Brady, M. \& Smith, S. Segmentation of Brain MR Images Through a Hidden Markov Random Field Model and the ExpectationMaximization Algorithm. leee T Med Imaging 20, 45-57 (2001).

79. Tournier, J., Calamante \& Connelly, F. \&. Improved probabilistic streamlines tractography by 2nd order integration over fibre orientation distributions. in Proc. 18th Annual Meeting of the Intl. Soc. Mag. Reson. Med.

80. Avants, B. B., Epstein, C. L., Grossman, M. \& Gee, J. C. Symmetric diffeomorphic image registration with cross-correlation: Evaluating automated labeling of elderly and neurodegenerative brain. Med Image Anal 12, 26-41 (2008).

81. Yeh, F.-C. et al. Population-averaged atlas of the macroscale human structural connectome and its network topology. Neuroimage 178, 5768 (2018).

82. Johnson, N. L., Kemp, A. W. \& Kortz, S. Univariate Discrete Distributions. (Wiley-Interscience, 1993).

83. Fonov, V. et al. Unbiased average age-appropriate atlases for pediatric studies. Neuroimage 54, 313-327 (2011).

84. Fonov, V., Evans, A., McKinstry, R., Almli, C. \& Collins, D. Unbiased nonlinear average age-appropriate brain templates from birth to adulthood. Neuroimage 47, S102 (2009).

85. Boatman, D., Lesser, R. P. \& Gordon, B. Auditory Speech Processing in the Left Temporal Lobe: An Electrical Interference Study. Brain Lang 51, 269-290 (1995).

86. Chang, E. F. et al. Categorical speech representation in human superior temporal gyrus. Nat Neurosci 13, 1428-1432 (2010).

87. Desai, R., Liebenthal, E., Waldron, E. \& Binder, J. R. Left Posterior Temporal Regions are Sensitive to Auditory Categorization. J Cognitive Neurosci 20, 1174-1188 (2008).

88. Rogalsky, C. et al. The Neuroanatomy of Speech Processing: A Large-Scale Lesion Study. Preprint at https://doi.org/10.1101/2020.04.02.022822 (2020).

89. Mirman, D. et al. Neural organization of spoken language revealed by lesion-symptom mapping. Nat Commun 6, 6762 (2015).

90. Wickens, D. D. Encoding categories of words: An empirical approach to meaning. Psychol Rev 77, 1-15 (1970).

91. Baddeley, A. The episodic buffer: a new component of working memory? Trends Cogn Sci 4, 417-423 (2000).

92. Prutting, C. A. \& Kittchner, D. M. A Clinical Appraisal of the Pragmatic Aspects of Language. J Speech Hear Disord 52, 105-119 (1987).

93. Yoshinaga-Itano, C., Sedey, A. L., Mason, C. A., Wiggin, M. \& Chung, W. Early Intervention, Parent Talk, and Pragmatic Language in Children With Hearing Loss. Pediatrics 146, S270-S277 (2020).

94. Calvert, G. A., Hansen, P. C., Iversen, S. D. \& Brammer, M. J. Detection of Audio-Visual Integration Sites in Humans by Application of Electrophysiological Criteria to the BOLD Effect. Neuroimage 14, 427-438 (2001).

95. Chen, T. \& Rao, R. R. Audio-visual integration in multimodal communication. P leee 86, 837-852 (1998).

96. Duffau, H., Gatignol, P., Moritz-Gasser, S. \& Mandonnet, E. Is the left uncinate fasciculus essential for language? J Neuro/ 256, 382-389 (2009).

97. Parker, G. J. M. et al. Lateralization of ventral and dorsal auditory-language pathways in the human brain. Neuroimage 24, 656-666 (2005).

98. Schotten, M. T. de, Dell'Acqua, F., Valabregue, R. \& Catani, M. Monkey to human comparative anatomy of the frontal lobe association tracts. Cortex 48, 82-96 (2012).

99. Arbel, Y. \& Donchin, E. Error and performance feedback processing by children with Specific Language Impairment-An ERP study. Biol Psychol 99, 83-91 (2014).

100. Mehl, M. R., Vazire, S., Ramírez-Esparza, N., Slatcher, R. B. \& Pennebaker, J. W. Are Women Really More Talkative Than Men? Science 317, 82-82 (2007). 
101. Demerens, C. et al. Induction of myelination in the central nervous system by electrical activity. Proc National Acad Sci $93,9887-9892$ (1996).

102. Ishibashi, T. et al. Astrocytes Promote Myelination in Response to Electrical Impulses. Neuron 49, 823-832 (2006).

103. Stevens, B., Porta, S., Haak, L. L., Gallo, V. \& Fields, R. D. Adenosine A Neuron-Glial Transmitter Promoting Myelination in the CNS in Response to Action Potentials. Neuron 36, 855-868 (2002).

104. Keller, T. A. \& Just, M. A. Altering Cortical Connectivity: Remediation-Induced Changes in the White Matter of Poor Readers. Neuron 64, 624631 (2009).

105. Averkin, R. G., Szemenyei, V., Bordé, S. \& Tamás, G. Identified Cellular Correlates of Neocortical Ripple and High-Gamma Oscillations during Spindles of Natural Sleep. Neuron 92, 916-928 (2016).

106. Bartos, M., Vida, I. \& Jonas, P. Synaptic mechanisms of synchronized gamma oscillations in inhibitory interneuron networks. Nat Rev Neurosci 8, 45-56 (2007).

107. Rangel, L. M. et al. Rhythmic coordination of hippocampal neurons during associative memory processing. Elife 5, e09849 (2016).

108. Suffczynski, P., Crone, N. E. \& Franaszczuk, P. J. Afferent inputs to cortical fast-spiking interneurons organize pyramidal cell network oscillations at high-gamma frequencies (60-200 Hz). J Neurophysiol 112, 3001-3011 (2014).

109. Varga, C. et al. Functional fission of parvalbumin interneuron classes during fast network events. Elife 3, e04006 (2014).

110. Whittington, M. A., Roopun, A. K., Traub, R. D. \& Davies, C. H. Circuits and brain rhythms in schizophrenia: a wealth of convergent targets. Curr Opin Pharmacol 11, 508-514 (2011).

111. Gaetz, W., Edgar, J. C., Wang, D. J. \& Roberts, T. P. L. Relating MEG measured motor cortical oscillations to resting Y-Aminobutyric acid (GABA) concentration. Neuroimage 55, 616-621 (2011).

112. Muthukumaraswamy, S. D., Edden, R. A. E., Jones, D. K., Swettenham, J. B. \& Singh, K. D. Resting GABA concentration predicts peak gamma frequency and fMRI amplitude in response to visual stimulation in humans. Proc National Acad Sci 106, 8356-8361 (2009).

113. Cousijn, H. et al. Resting GABA and glutamate concentrations do not predict visual gamma frequency or amplitude. Proc National Acad Sci 111, 9301-9306 (2014).

114. Arya, R. et al. Development of information sharing in language neocortex in childhood-onset drug-resistant epilepsy. Epilepsia 60, 393-405 (2019).

115. Klooster, M. A. van 't et al. Time-frequency analysis of single pulse electrical stimulation to assist delineation of epileptogenic cortex. Brain 134, 2855-2866 (2011).

116. Hallett, M. et al. Contribution of transcranial magnetic stimulation to assessment of brain connectivity and networks. Clin Neurophysiol 128, 2125-2139 (2017).

117. Ziemann, U., Lönnecker, S., Steinhoff, B. J. \& Paulus, W. Effects of antiepileptic drugs on motor cortex excitability in humans: A transcranial magnetic stimulation study. Ann Neurol 40, 367-378 (1996).

118. Borah, N. C. \& Matheshwari, M. C. Effect of antiepileptic drugs on short-latency somatosensory evoked potentials. Acta Neurol Scand 71, 331-333 (1985).

119. Centeno, M. \& Carmichael, D. W. Network Connectivity in Epilepsy: Resting State fMRI and EEG-fMRI Contributions. Front Neuro/ 5, 93 (2014).

120. Dansereau, C. L. et al. Detection of abnormal resting-state networks in individual patients suffering from focal epilepsy: an initial step toward individual connectivity assessment. Front Neurosci-switz 8, 419 (2014).

121. Lee, H. W. et al. Altered functional connectivity in seizure onset zones revealed by fMRI intrinsic connectivity. Neurology 83 , 2269-2277 (2014).

\section{Figures}




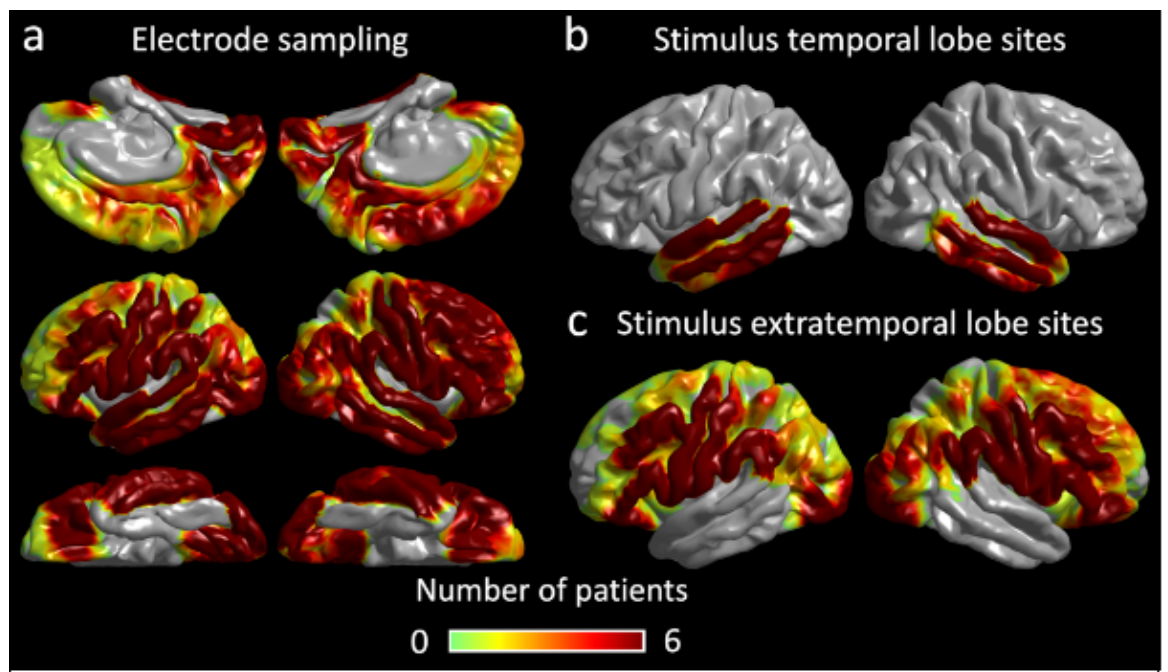

Fig.1|Spatial distribution of subdural electrode sampling. a, The surface image presents the spatial distribution of analyzed electrodes on each hemisphere. Color indicates the number of patients available at a given spatial point. A total of 3,401 non-epileptic artifact-free electrode sites were included in the analysis. $\mathbf{b}$, The spatial distribution of 488 stimulus sites in the temporal lobe used for the assessment of CCSR-based temporal to extratemporal lobe connectivity. c, The spatial distribution of 1,581 stimulus sites in the extratemporal lobe used for the assessment of the connectivity in the opposite direction.

Figure 1

(Caption included in graphic)

\section{a Auditory naming task}

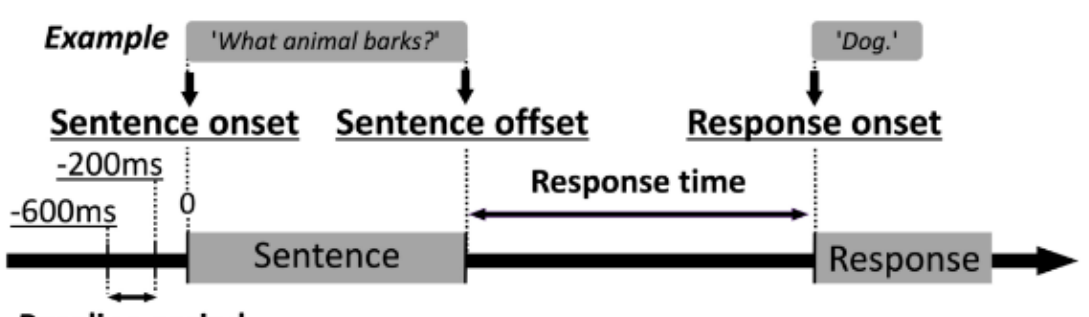

Baseline period

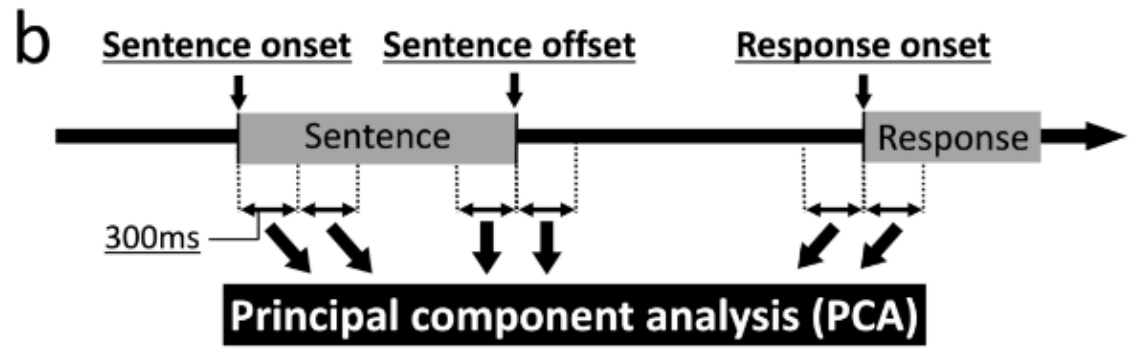

Fig.2|Auditory naming task and the time windows incorporated in the principal component analysis (PCA). a, Patients were instructed to give overt verbal answers to each of the auditory sentence questions (median duration of question sentence: $1.8 \mathrm{~s}$; range: 1.2 to $2.4 \mathrm{~s}$ ). Naming-related high-gamma activity during each $300 \mathrm{~ms}$ window was normalized as a relative value to the pre-trial baseline. $\mathbf{b}$, To determine what linguistic stages a given electrode channel was involved in, we employed a PCA to high-gamma modulations during six 300 ms time windows, that include three 300 ms periods during stimulus listening, two $300 \mathrm{~ms}$ periods between stimulus offs et and response onset, as well as a $300 \mathrm{~ms}$ period immediately after overt response. 


\section{Figure 2}

(Caption included in graphic)
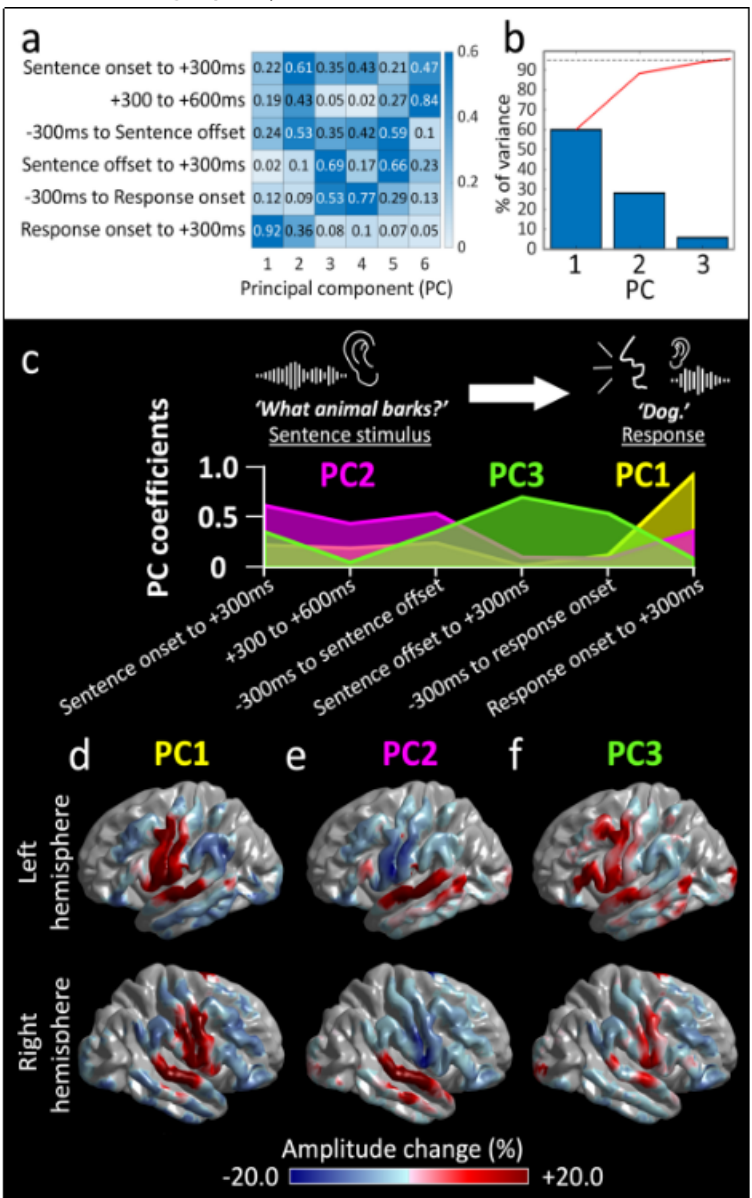

Fig.3 | Linguistic stage categorization based on a PCA of naming-related high gamma modulations. a, This heat map presents the contributions (as reflected by the principal component coefficients) of naming-related high-gamma modulation during each $300 \mathrm{~ms}$ window to the principal components (PCs). b, The first 3 PCs account for approximately $95 \%$ of the variance (broken line) in high-gamma temporal patterns (red line). c, Relative contribution of PCs 1-3 to each time window. d-f, The spatial distribution of each PC. A color is displayed at sites where $\geq 4$ patients' data were available.

\section{Figure 3}

(Caption included in graphic) 


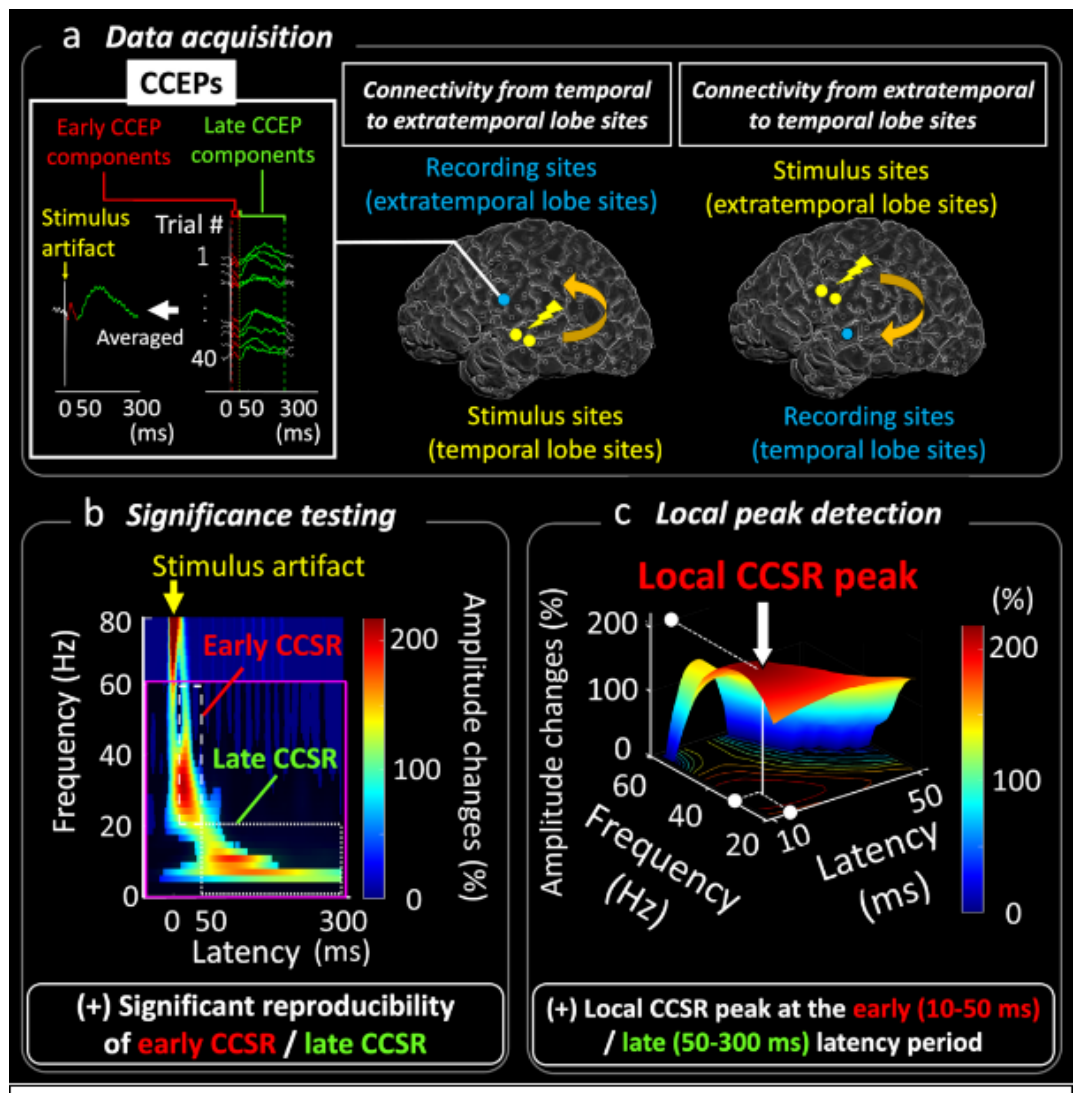

Fig.4| Workflow to quantify inter-lobar effective connectivity based on cortico-cortical spectral responses (CCSRs). a, CCEPs: Averaged ECoG trace and single-trial ECoG traces are aligned to the onset of single-pulse electrical stimulation. $\mathbf{b}$, To identify significant CCSRs, a time-frequency transformation followed by a cluster-based permutation test was employed for the 2 to $60 \mathrm{~Hz}$ and -50 to $+300 \mathrm{~ms}$ time-frequency range (pink square) ${ }^{74}$. [1] Early CCSR: within 20 to $60 \mathrm{~Hz}$ and +10 and $+50 \mathrm{~ms}$ (white dashed square $)^{47,67}$. This time-frequency range was largely free of stimulation-related broadband artifacts (yellow arrow). [2] Late CCSR: within 2 and $20 \mathrm{~Hz}$ and +50 and $+300 \mathrm{~ms}$ (white dotted square). c, Early CCSR peaks were taken as evidence of $\underline{\text { direct }}$ effective connectivity; late CCSRs peaks were taken as evidence of indirect effective connectivity.

Figure 4

(Caption included in graphic) 


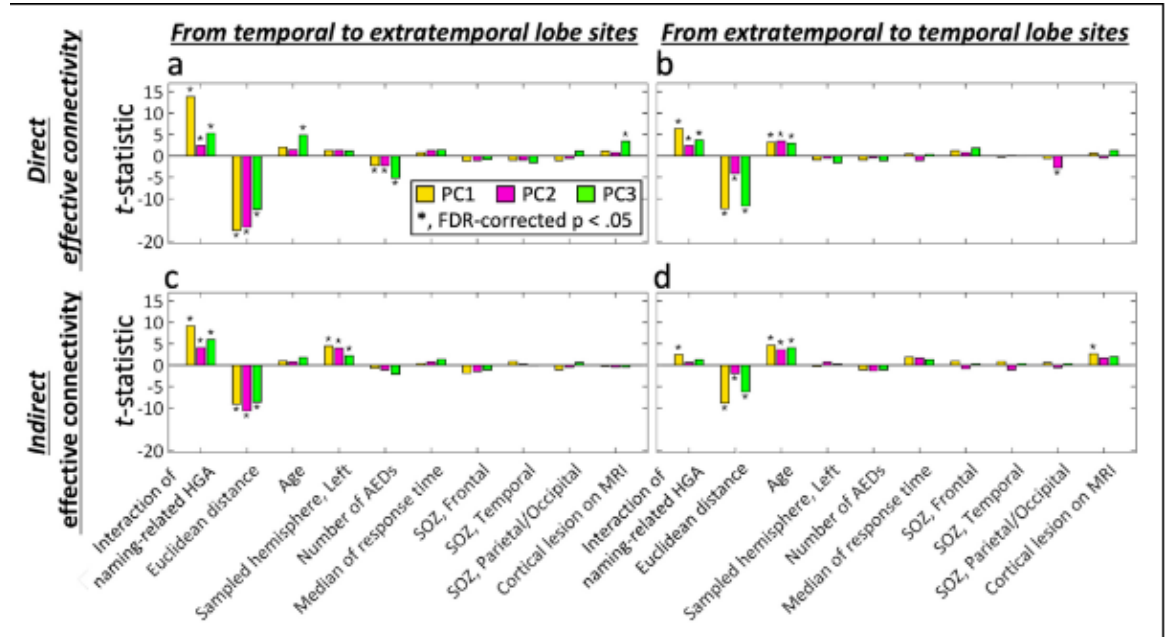

Fig.5 | Mixed model-based prediction of the strength of direct and indirect effective connectivity.

The bar graphs show the effects of variables for predicting the strength of (a-b) direct and $(\mathbf{c}-\mathbf{d})$ indirect inter-lobar effective connectivity between language sites supporting the same linguistic stages. a and c, Prediction of effective connectivity in the temporal to extratemporal lobe direction. $\mathbf{b}$ and $\mathbf{d}$, Connectivity in the opposite direction. Bars indicate the effect of predictors in each PC-define network: Yellow bars: PC1. Pink bars: PC2. Green bars: PC3. *: FDR-corrected p-value < 0.05. PC: principal component.

\section{Figure 5}

(Caption included in graphic) 


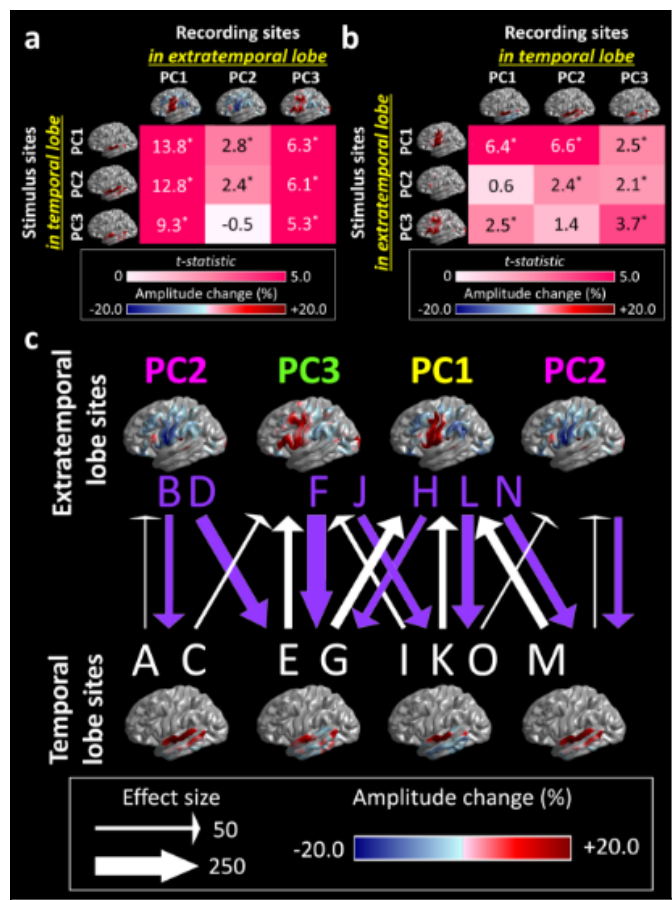

Fig $6 \mid$ Direct inter-lobar effective connectivity across sites supporting the same and different linguistic stages. a-b, The statistical effect (i.e., mixed model t-values) of naming-related high-gamma activity (HGA) interaction between sites supporting the same and different principal component $(\mathrm{PC})$ language sites on the strength of direct inter-lobar effective connectivity. *: FDR-corrected $p<0.05$. c, The effect size (i.e., significant mixed model estimate value) of naming-related HGA interaction on the strength of direct inter-lobar effective connectivity is indicated by the arrowhead length and the arrow width (A-N). Connectivity direction is presented by arrow color: white, a temporal-to-extratemporal direction; purple, the opposite direction. For example, effective connectivity to PC3 extratemporal language sites was the strongest from the PC3 temporal lobe sites (white arrow $\mathbf{E}$; effect size: $+106.1 \%$ ) while smallfrom the PC1 (white arrow I; effect size: $+61.0 \%$ ) and PC2 (white arrow C; effect size: $+48.2 \%$ ). Effective connectivity to $\mathrm{PC} 3$ temporal lobe sites was strongest from $\mathrm{PC} 3$ extra-temporal lobe sites (purple arrow F; effect size: $+254.3 \%$ ) while moderate from PC1 (purple arrow $\mathbf{H}$; effect size: $+99.5 \%$ ) and PC2 (purple arrow D; effect size: $+169.4 \%$ ).

\section{Figure 6}

(Caption included in graphic) 


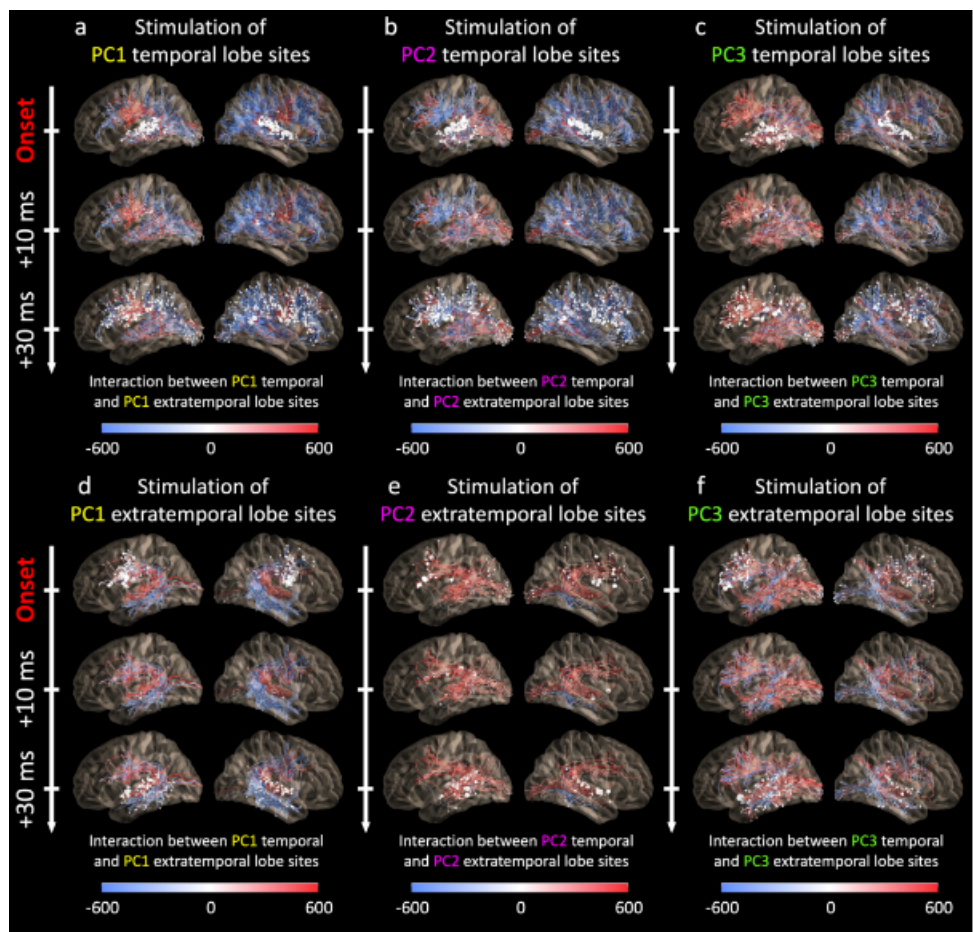

Fig. 7 |Snapshots of 6D dynamic tractography. The snapshots of each animation demonstrate the trajectories of rapid neural propagations elicited by single-pul se electrical stimulation and its relationship to naming-related high-gamma modulations at the stimulus and recording sites. Each plot displays grouplevel effective connectivity data derived from 27 patients. The streamline color reflects the interaction (i.e., multiplication) of high-gamma activity ( $\mathrm{HGA}$ ) at temporal and extratemporal lobe sites during a given linguistic stage. Thus, red streamlines reflect high-gamma co-augmentation at both stimulus and recording sites, whereas blue ones indicate high-gamma augmentation at the stimulus sites but attenuation at the recording sites. The size of each white dot reflects the strength of effective connectivity rated by the magnitude of significant early cortico-cortical spectral response (CCSR) local peak. Each white dot indicates the location of stimulation-induced neural propagation es timated by the propagation velocity. To optimize visibility, each movie delineates the effective connectivity pathways satisfying the following criteria: [a] stimulus sites with naming-related high-gamma augmentation during a specific PCA-based linguistic stage, [b] the interaction of naming-related HGA was $\geq| \pm 200|$, and [c] a given strea mline was associated with a significant early CCSR. a-c, Temporal to extratemporal lobe propagations (See Video S1). d-f, Extratemporal to temporal lobe propagations (See Video S2). a and d, Neural propagations elicited by stimulation of PC1 language sites. b and e, $\mathrm{PC2} . \mathrm{c}$ and $\mathrm{f}, \mathrm{PC}$.

\section{Figure 7}

(Caption included in graphic)

\section{Supplementary Files}

This is a list of supplementary files associated with this preprint. Click to download.

- VideoS1.mp4

- VideoS2.mp4

- Onlinesupplementarydocumentms37ea13bhs1.docx 